\title{
An Alkali Metal-Capped Cerium(IV) Imido Complex
}

Lukman A. Solola, Alexander V. Zabula, Walter L. Dorfner, Brian C. Manor, Patrick J. Carroll and Eric J. Schelter*

P. Roy and Diana T. Vagelos Laboratories, Department of Chemistry, University of Pennsylvania, 231 S $34^{\text {th }}$ St., Philadelphia, Pennsylvania 19104, United States

E-mail: schelter@sas.upenn.edu

Supporting Information

$\begin{array}{ll}\text { Figure S1. Thermal ellipsoid plot for } 2 & \text { S2 }\end{array}$

Figure S2. UV-vis spectra for 2-4 measured in THF $\quad$ S2

Figure S3. Fit of the UV-vis spectrum for 4 and corresponding FWHM's $\quad$ S3

$\begin{array}{ll}\text { Synthetic Details and Characterization } & \text { S3 }\end{array}$

General Methods $\quad$ S3

$\begin{array}{ll}\text { Materials } & \mathrm{S} 3 \\ \end{array}$

Data for Ce(TriNOx)(N(SiHMe $\left.)_{2}\right)(2) \quad$ S4

Figure S4. ${ }^{1} \mathrm{H}$ NMR spectrum for $\mathbf{2}$ S5

Figure S5. ${ }^{13} \mathrm{C}$ NMR spectrum for $2 \quad \mathrm{~S} 5$

Data for Ce(TriNOx) $\left[\mathrm{NH}\left(3,5-\left(\mathrm{CF}_{3}\right)_{2} \mathbf{C}_{6} \mathrm{H}_{3}\right)\right](3) \quad$ S6

Figure S6. ${ }^{1} \mathrm{H}$ NMR spectrum for $\mathbf{3}$ S6

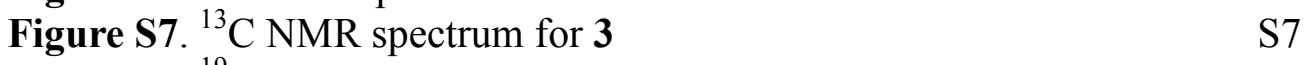

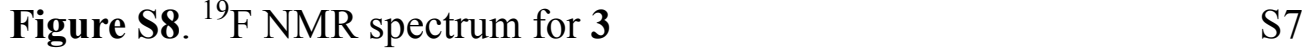

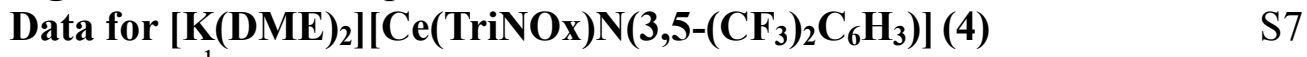

Figure S9. ${ }^{1} \mathrm{H}$ NMR spectrum for $4 \quad$ S8

Figure S10. ${ }^{13} \mathrm{C}$ NMR spectrum for $4 \quad$ S9

Figure S11. ${ }^{19} \mathrm{~F}$ NMR spectrum for $4 \quad$ S9

Data for Ce(TriNOx)[N(SiMe 3$\left.)\left(3,5-\left(\mathrm{CF}_{3}\right)_{2} \mathrm{C}_{6} \mathrm{H}_{3}\right)\right](5) \quad \mathrm{S} 9$

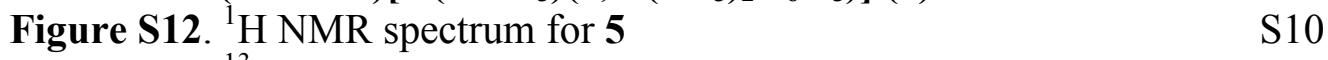

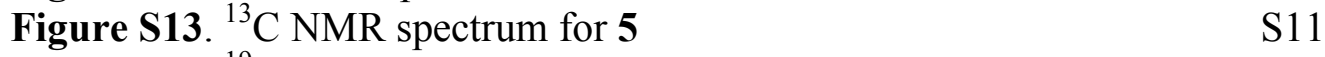

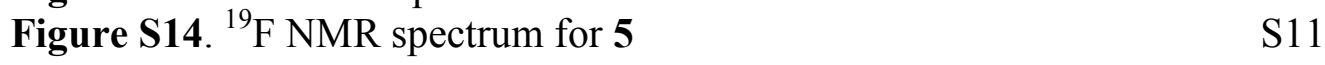

$\begin{array}{ll}\text { Figure S15. UV-vis spectrum for } 5 & \text { S12 }\end{array}$

$\begin{array}{ll}\text { Crystal Structure Determination and Refinement } & \text { S12 }\end{array}$

Table S1. Crystallographic Data for 2, 3·THF, 4, and $5 \quad$ S13

$\begin{array}{ll}\text { Computation Details } & \text { S14 }\end{array}$

$\begin{array}{ll}\text { Table S2. Absolute energies for } \mathbf{3}, \mathbf{4} \text { and } \mathbf{4}^{-} & \text {S14 }\end{array}$

Table S3. Cartesian coordinates for $3 \quad$ S14

$\begin{array}{ll}\text { Table S4. Cartesian coordinates for } 4 & \text { S17 }\end{array}$

$\begin{array}{ll}\text { Table S5. Cartesian coordinates for } \mathbf{4}^{-} & \text {S21 }\end{array}$

References

S24 

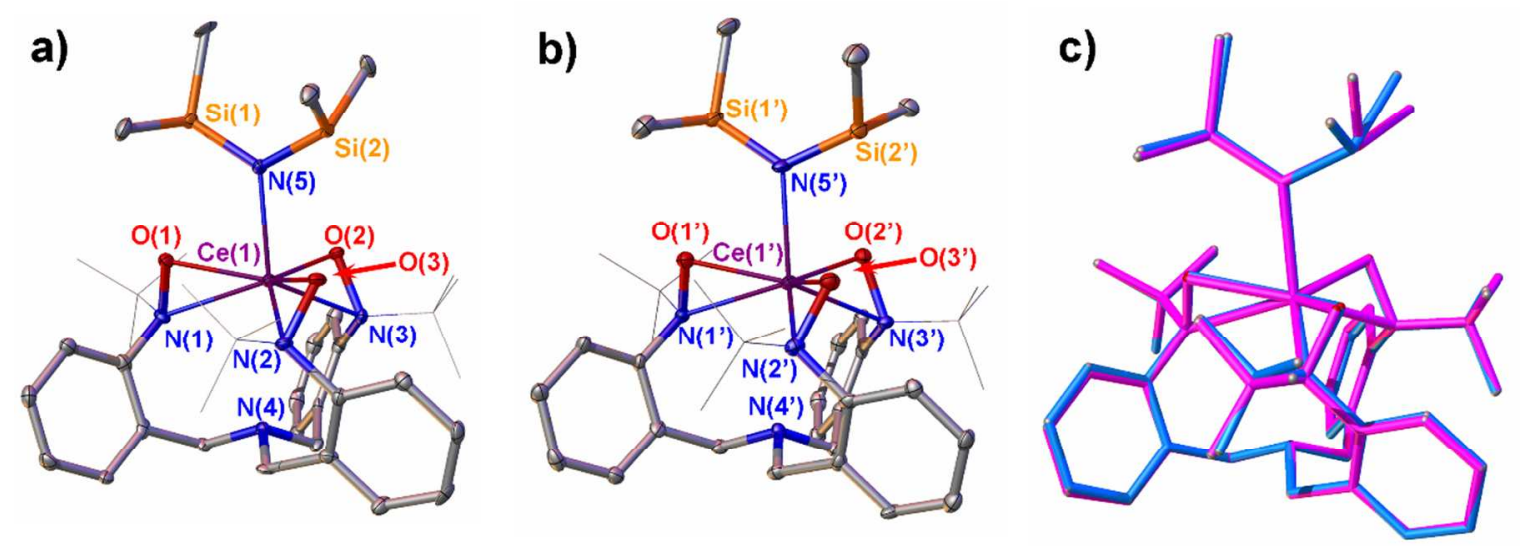

Figure S1. Thermal ellipsoid plot for two crystallographically independent molecules of 2 (2A (a) and 2B (b)) shown at the 30\% probability level and a stick model of their superposition (c). Hydrogen atoms have been omitted for clarity. $t$-Butyl groups are depicted using a wire model (a and $\mathbf{b})$. Selected bond distances $(\AA)$ and angles $\left(^{\circ}\right)$ for $\mathbf{2 A}$ (the related geometrical parameters for $\mathbf{2} \mathbf{B}$ are given in square brackets): $\mathrm{Ce}(1)-\mathrm{O}(1)$ 2.167(4) [2.179(4)], $\mathrm{Ce}(1)-\mathrm{O}(2)$ 2.193(4) [2.169(5)], Ce(1)-O(3) 2.191(4) [2.176(4)], $\mathrm{Ce}(1)-\mathrm{N}(1)$ 2.577(5) [2.598(6)], $\mathrm{Ce}(1)-\mathrm{N}(2)$ 2.551(5) [2.570(5)], Ce(1)-N(3) 2.581(5) [2.548(5)], $\mathrm{Ce}(1)-\mathrm{N}(5)$ 2.353(5) [2.370(5)]; $\mathrm{Ce}(1)-\mathrm{N}(5)-\mathrm{Si}(1)$ 126.2(2) [111.8(3)], $\mathrm{Ce}(1)-$ $\mathrm{N}(5)-\operatorname{Si}(2)$ 113.7(3) [125.9(3)].

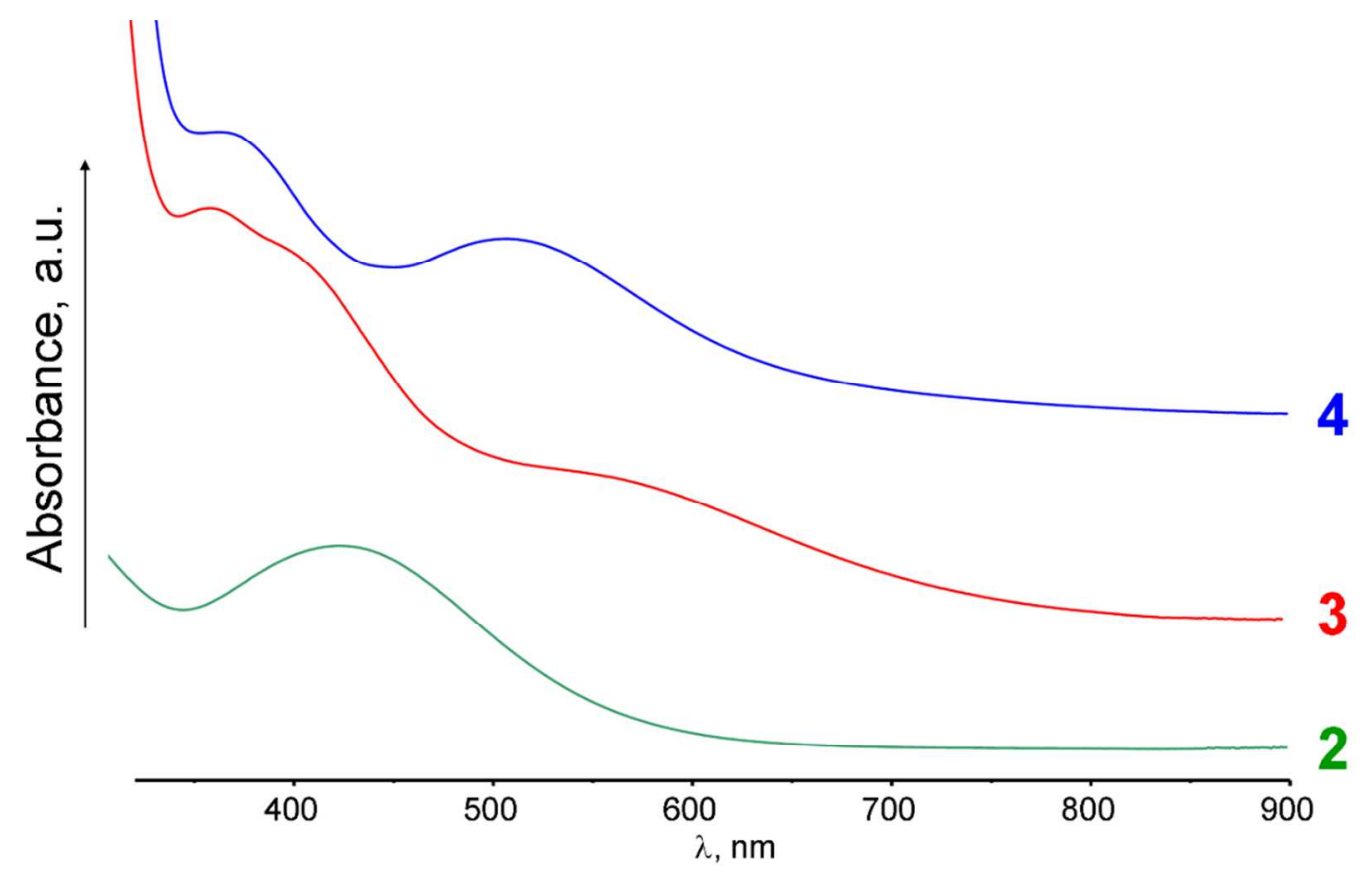

Figure S2. UV-vis spectra for $\mathbf{2}-\mathbf{4}$ measured in THF. 


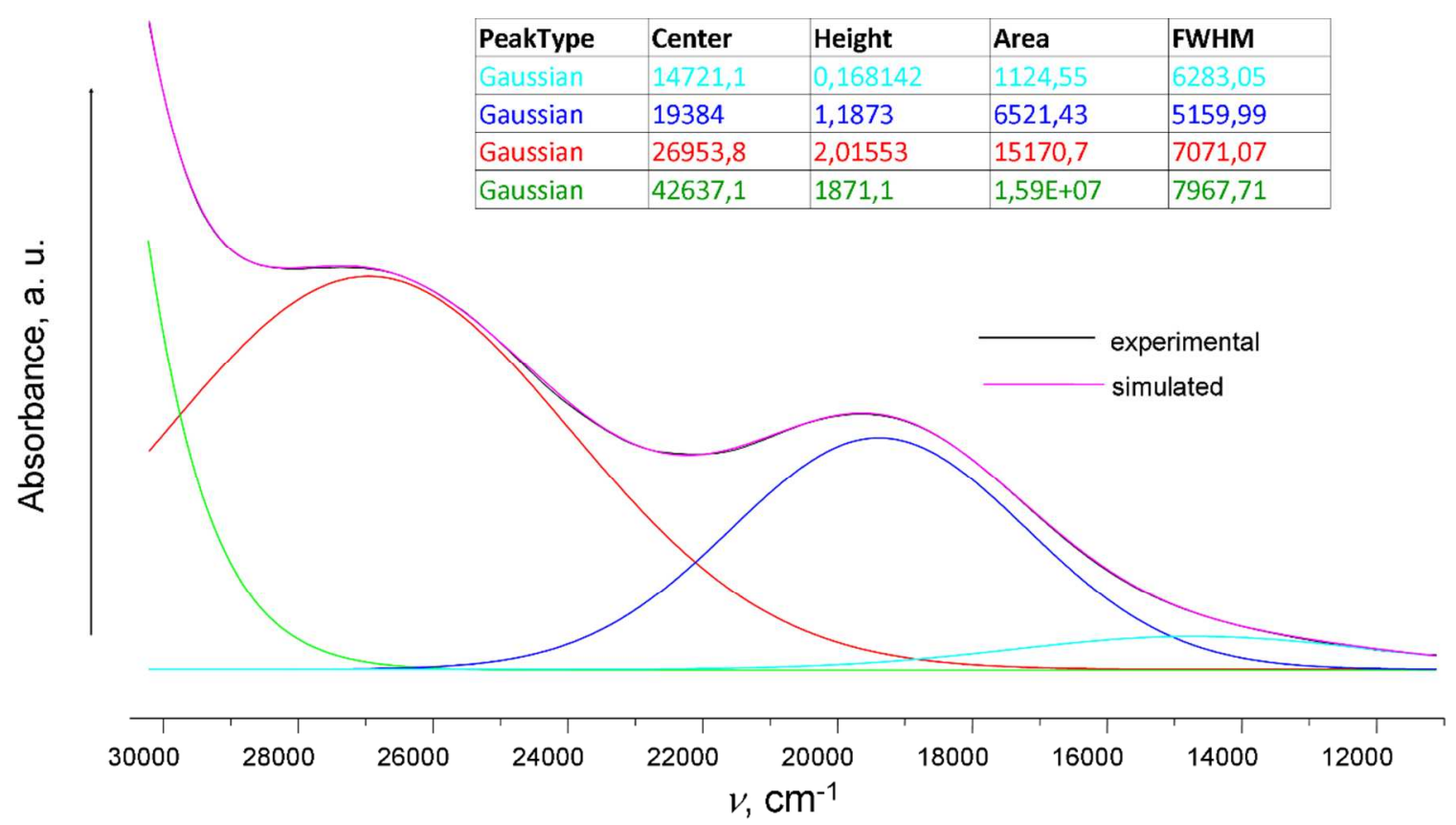

Figure S3. Fit of the UV-vis spectrum for 4 and corresponding FWHM's.

\section{Synthetic Details and Characterization}

General Methods. All reactions and manipulations were performed under an inert atmosphere $\left(\mathrm{N}_{2}\right)$ using standard Schlenk techniques or in a drybox equipped with a molecular sieves 13X/Q5 Cu-0226S catalyst purifier system. Glassware was oven-dried for at least $3 \mathrm{~h}$ at $150{ }^{\circ} \mathrm{C}$ prior to use. ${ }^{1} \mathrm{H},{ }^{13} \mathrm{C}\left\{{ }^{1} \mathrm{H}\right\}$ and ${ }^{19} \mathrm{~F}$ NMR spectra were obtained on a Bruker DMX-300 Fourier transform NMR spectrometer operating at $300 \mathrm{MHz}$ for ${ }^{1} \mathrm{H}$, 75.48 MHz for ${ }^{13} \mathrm{C}$ and $282.2 \mathrm{MHz}$ for ${ }^{19} \mathrm{~F} .{ }^{13} \mathrm{C}\left\{{ }^{1} \mathrm{H}\right\}$ NMR spectra for complexes $\mathbf{3}, \mathbf{4}$ and 5 were measured on Bruker UNI500 spectrometer working at $125.77 \mathrm{MHz}$ for ${ }^{13} \mathrm{C}$. Chemical shifts were recorded in units of parts per million and referenced against residual proteo solvent peaks $\left({ }^{1} \mathrm{H}\right)$, characteristic solvent peaks $\left({ }^{13} \mathrm{C}\right)$ or $\mathrm{CFCl}_{3}$ standard $\left({ }^{19} \mathrm{~F}\right)$. Infrared spectra were measured on a PerkinElmer 1600 series spectrometer. UV-vis spectra were recorded on PerkinElmer Lamba 950 spectrometer. Elemental analyses were performed at Midwest Microlab, Inc (Indianapolis, Indiana).

Materials. THF, DME, diethyl ether, fluorobenzene, and hexanes were sparged for 20 min with dry argon and dried using a commercial two-column solvent purification system comprising columns packed with Q5 reactant and neutral alumina respectively (for 
hexanes). Deuterated chloroform (Cambridge Isotopes) was degassed and stored over molecular sieves ( $4 \AA$ ) overnight prior to use. $\mathrm{C}_{6} \mathrm{D}_{6}$ (Cambridge Isotopes) was dried over a potassium mirror prior to use. THF- $d_{8}$ was dried over sodium-potassium alloy and distilled prior to use. Potassium bis(trimethylsilyl)amide (Sigma Aldrich) was used as received. Iodine (Alfa Aesar) was sublimed before use. 3,5-Bis(trifluoromethyl)aniline (Oakwood Chemicals) and $\left(\mathrm{CH}_{3}\right)_{3} \mathrm{SiCl}$ (Acros Chemicals) were distilled and dried over 4 $\AA$ sieves before use. $[\mathrm{Ce}(\mathrm{TriNOx})(\mathrm{THF})]^{1}$ and $\mathrm{KN}\left(\mathrm{SiHMe}_{2}\right)_{2}{ }^{2}$ were prepared according to published reports.

Data for Ce(TriNOx)(N(SiHMe $\left.)_{2}\right)$ (2). To a yellow suspension of [Ce(TriNOx)(THF)] (1) $(371 \mathrm{mg}, 0.49 \mathrm{mmol}, 1.0$ equiv) in $4 \mathrm{~mL}$ diethyl ether was added solid iodine (63 $\mathrm{mg}$, $0.25 \mathrm{mmol}, 0.5$ equiv) and the brown mixture was stirred for $2 \mathrm{~h}$. A solution of $\mathrm{KN}\left(\mathrm{SiHMe}_{2}\right)_{2}(84 \mathrm{mg}, 0.49 \mathrm{mmol}, 1.0$ equiv) in $4 \mathrm{~mL}$ of diethyl ether was then added and the mixture was stirred for additional $3 \mathrm{~h}$. Precipitated KI was removed by filtration over a Celite-packed coarse porosity fritted filter and the solvent was removed under reduced pressure from the filtrate. The resulting red solid product was dried in vacuo and recrystallized upon cooling a hot hexanes solution to $-25^{\circ} \mathrm{C}$. Yield $100 \mathrm{mg}, 25 \%$.

${ }^{1} \mathrm{H}$ NMR $\left(300 \mathrm{MHz}, \mathrm{C}_{6} \mathrm{D}_{6}\right): \delta 7.47\left(\mathrm{~d},{ }^{2} J_{\mathrm{HH}}=8.2 \mathrm{~Hz}, 3 \mathrm{H}, \mathrm{Ar}-\mathrm{H}\right), 7.07-7.024(\mathrm{~m}, 3 \mathrm{H}, \mathrm{Ar}-$ H), 6.95-6.91 (m, 6H, Ar-H), 6.71 (septet, $\left.{ }^{2} J_{\mathrm{HH}}=3.4 \mathrm{~Hz}, 2 \mathrm{H}, \mathrm{SiH}\right), 4.46\left(\mathrm{~d},{ }^{1} J_{\mathrm{HH}}=11.7\right.$ $\left.\mathrm{Hz}, 3 \mathrm{H}, \mathrm{CH}_{2}\right), 2.34\left(\mathrm{~d},{ }^{1} J_{\mathrm{HH}}=11.7 \mathrm{~Hz}, 3 \mathrm{H}, \mathrm{CH}_{2}\right), 0.89\left(\mathrm{~d},{ }^{2} J_{\mathrm{HH}}=3.4 \mathrm{~Hz}, 6 \mathrm{H}, \mathrm{SiH}\left(\mathrm{CH}_{3}\right)_{2}\right)$, $0.86\left(\mathrm{~d},{ }^{2} J_{\mathrm{HH}}=3.4 \mathrm{~Hz}, 6 \mathrm{H}, \mathrm{SiH}\left(\mathrm{CH}_{3}\right)_{2}\right), 0.84\left(\mathrm{~s}, 27 \mathrm{H}, \mathrm{C}\left(\mathrm{CH}_{3}\right)_{3}\right) .{ }^{13} \mathrm{C}$ NMR $(75.48 \mathrm{MHz}$, $\left.\mathrm{C}_{6} \mathrm{D}_{6}\right): \delta$ 150.06, 132.47, 131.43, 129.42, 128.37, 126.13 (Ar-C), $64.89\left(\mathrm{CH}_{2}\right), 60.99$

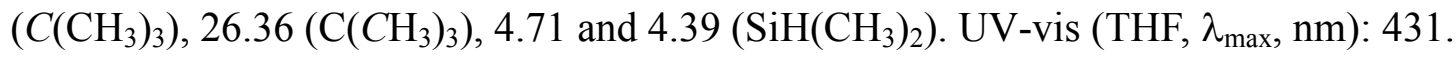
Anal. Calc'd for $\mathrm{C}_{37} \mathrm{H}_{59} \mathrm{CeN}_{5} \mathrm{O}_{3} \mathrm{Si}_{2}$ : C, 54.31; H, 7.27; N, 8.56. Found: C, 54.40; H, 6.97; N, 8.32 . 


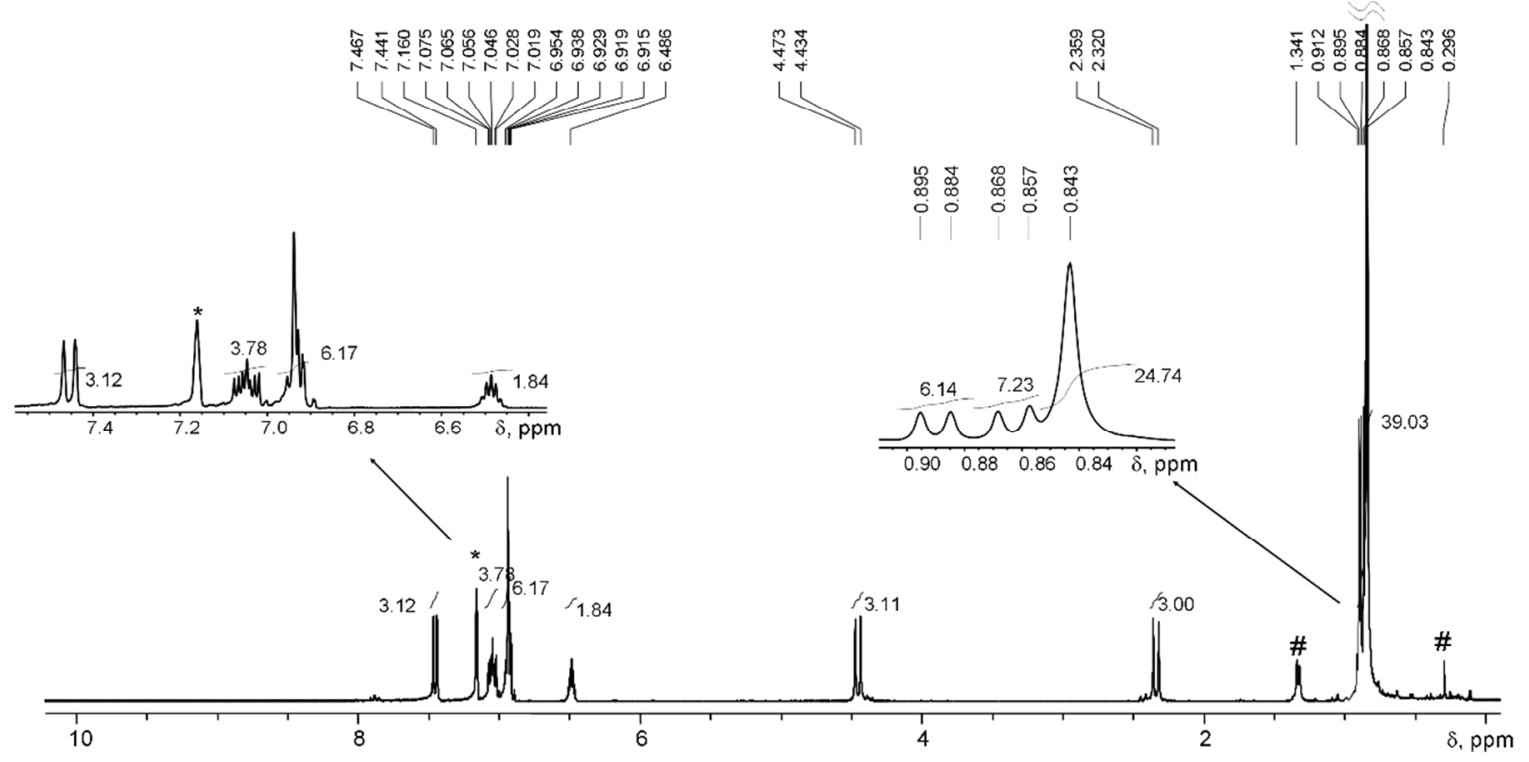

Figure S4. ${ }^{1} \mathrm{H}$ NMR spectrum for 2 (in $\mathrm{C}_{6} \mathrm{D}_{6},{ }^{*}$ - proteo solvent signal, \# - grease).

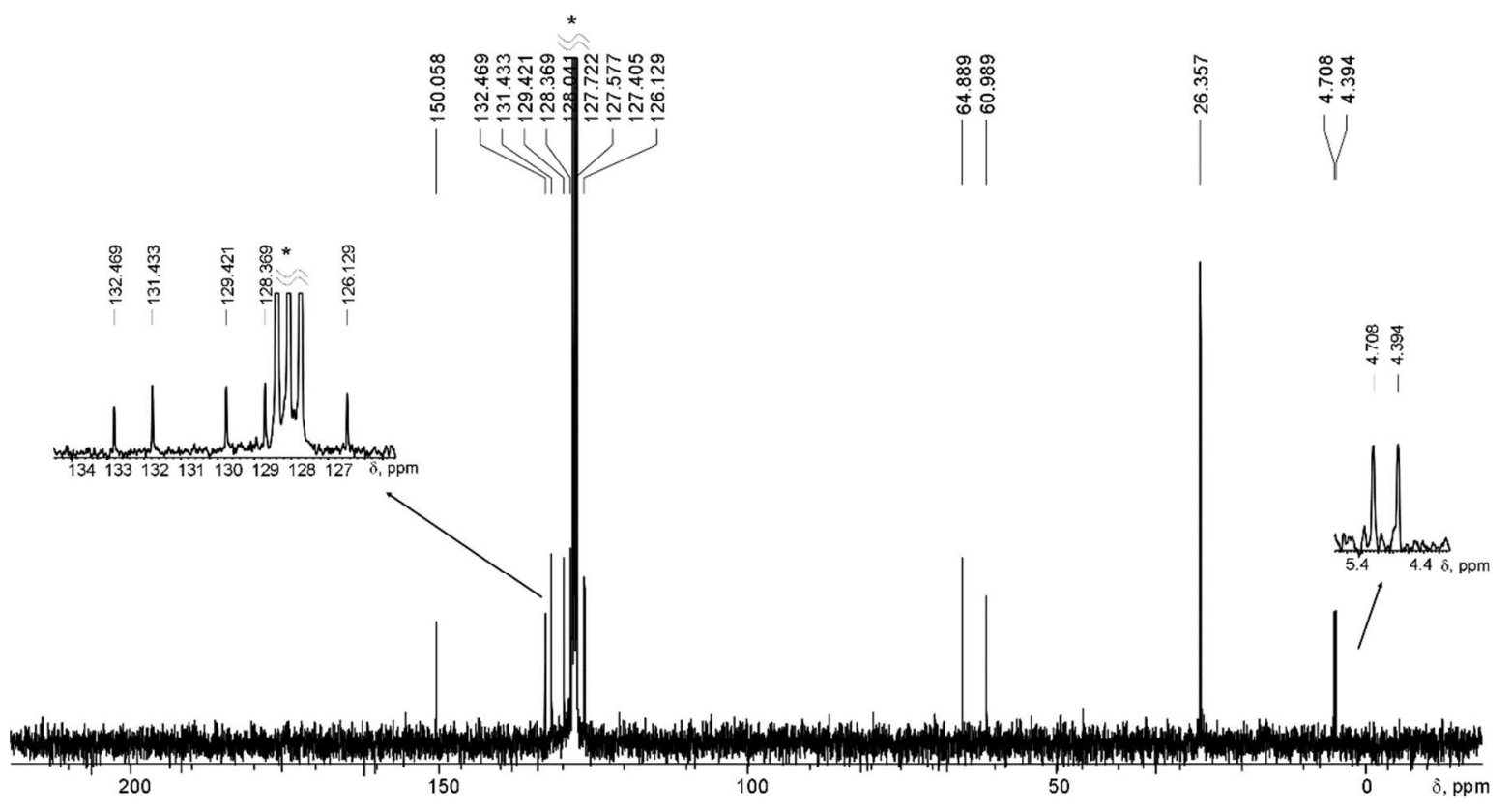

Figure S5. ${ }^{13} \mathrm{C}$ NMR spectrum for 2 (in $\mathrm{C}_{6} \mathrm{D}_{6}, *$ - solvent signal).

Data for Ce(TriNOx) $\left[\mathbf{N H}\left(3,5-\left(\mathbf{C F}_{3}\right)_{2} \mathbf{C}_{6} \mathbf{H}_{3}\right)\right]$ (3). A solution of $\mathrm{H}_{2} \mathrm{~N}-\left(3,5-\left(\mathrm{CF}_{3}\right)_{2} \mathrm{C}_{6} \mathrm{H}_{3}\right)$

( $161 \mathrm{mg}, 0.70 \mathrm{mmol}, 1.5$ equiv) in $4 \mathrm{~mL}$ of $n$-pentane was added to solid 2 (382 $\mathrm{mg}, 0.47$ mmol, 1.0 equiv) and the resulting suspension was stirred for $12 \mathrm{~h}$. The reaction mixture was then filtered over a medium porosity fritted filter. The formed black solid complex 3 
was washed with $n$-pentane $(3 \times 20 \mathrm{~mL})$ and dried under reduced pressure. Yield $398 \mathrm{mg}$, 93\%. X-ray quality crystals of $3 \cdot$ THF were grown from a hot THF solution.

${ }^{1} \mathrm{H}$ NMR (300 MHz, $\mathrm{CDCl}_{3}$ ): $\delta$ 7.46-7.37 (m, 9H, Ar-H), 7.31-7.24 (m, 6H, Ar-H), 6.57 (s, NH, 1H), $4.55\left(\mathrm{~d},{ }^{1} J_{\mathrm{HH}}=11.9 \mathrm{~Hz}, \mathrm{CH}_{2}, 3 \mathrm{H}\right), 2.85\left(\mathrm{~d},{ }^{1} J_{\mathrm{HH}}=11.9 \mathrm{~Hz}, \mathrm{CH}_{2}, 3 \mathrm{H}\right), 0.78$ (s, $\left.27 \mathrm{H}, \mathrm{CH}_{3}\right) .{ }^{13} \mathrm{C}$ NMR $\left(125.77 \mathrm{MHz}\right.$, THF- $\left.d_{8}\right): 161.76,149.47,133.34,133.00,130.96$ $\left(\mathrm{q}, J_{\mathrm{FC}}=30.5 \mathrm{~Hz}\right), 130.61,129.86,128.05,126.16\left(\mathrm{q}, J_{\mathrm{FC}}=272.6 \mathrm{~Hz}\right), 116.56\left(\mathrm{dd}, J_{\mathrm{FC}}=\right.$

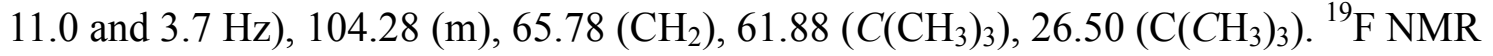
(282.2 MHz, $\mathrm{CDCl}_{3}$ ): $\delta-62.92$ (s). UV-vis (THF, $\lambda_{\max }, \mathrm{nm}$ ): 361, 402, 581. IR (KBr, $\mathrm{cm}^{-}$ $\left.{ }^{1}\right)$ : $3311(\mathrm{~m}, v(\mathrm{~N}-\mathrm{H}))$. Anal. Calc'd for $\mathrm{C}_{41} \mathrm{H}_{49} \mathrm{CeF}_{6} \mathrm{~N}_{5} \mathrm{O}_{3}$ : C, 53.88; H, 5.40; N, 7.66.

Found: C, 53.68; H, 5.30; N, 7.30.

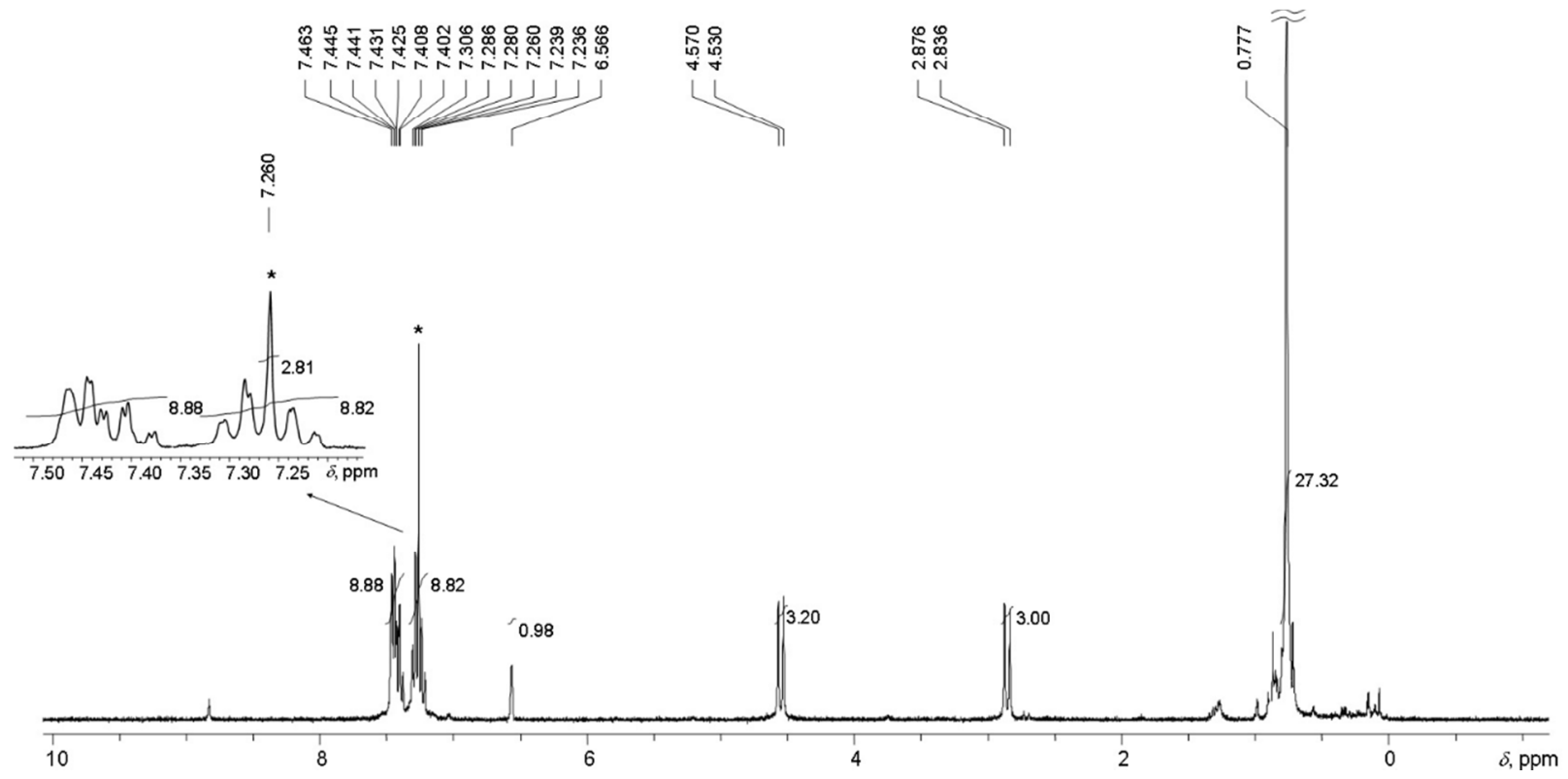

Figure S6. ${ }^{1} \mathrm{H}$ NMR spectrum for 3 (in $\mathrm{CDCl}_{3}$, * - proteo solvent signal). 


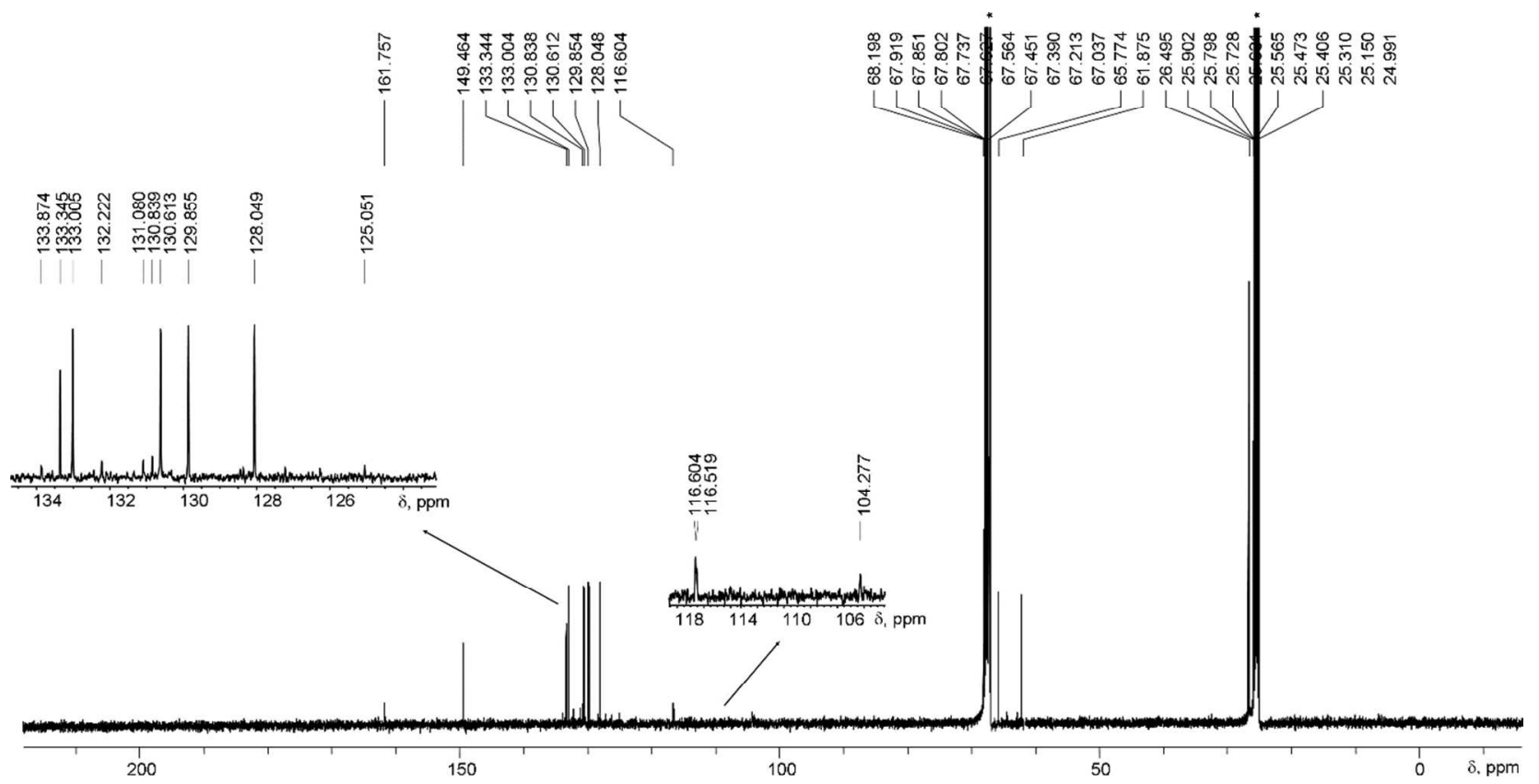

Figure S7. ${ }^{13} \mathrm{C}$ NMR spectrum for 3 (in THF- $d_{8},{ }^{*}$ - solvent signal).

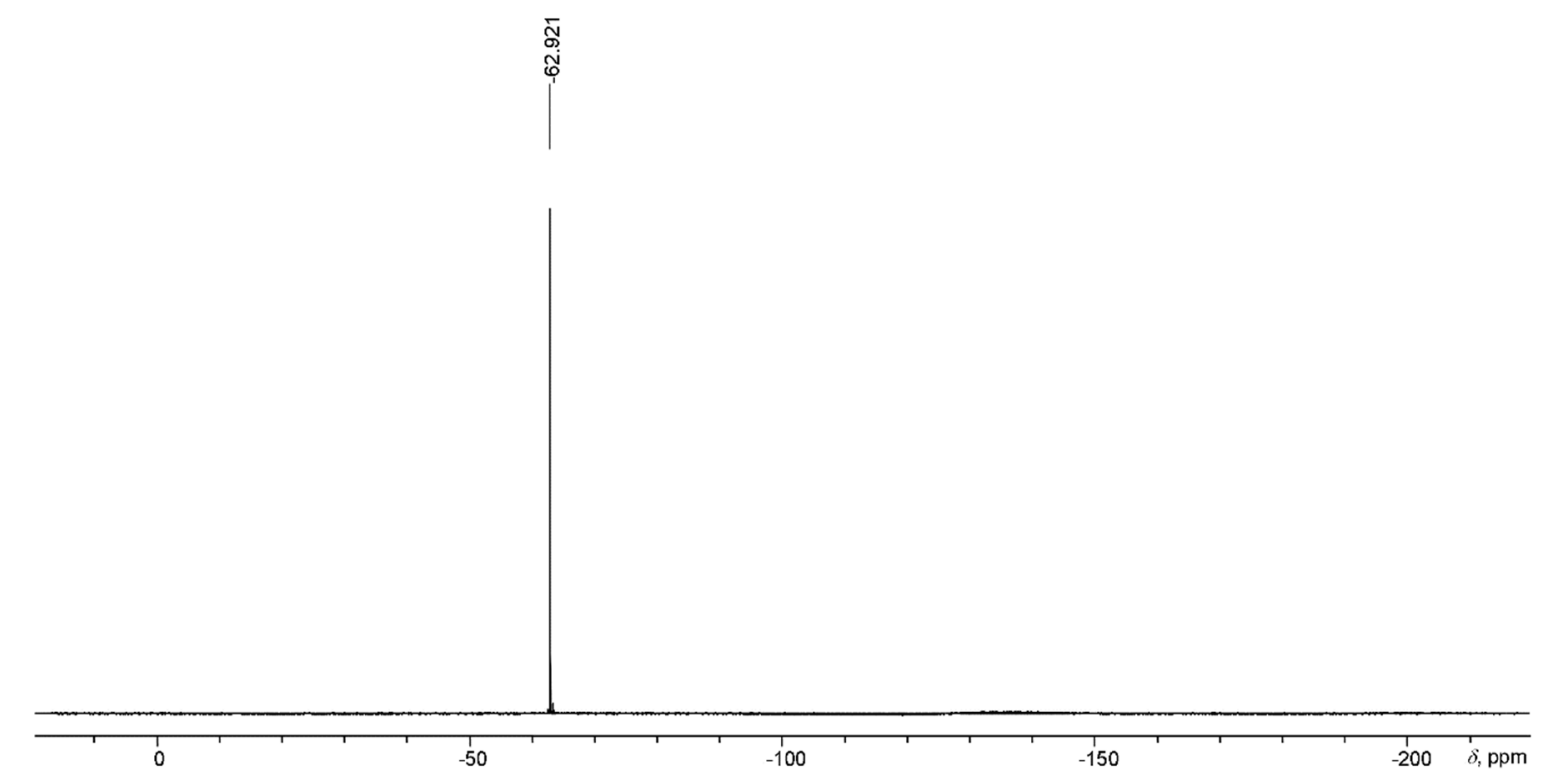

Figure S8. ${ }^{19} \mathrm{~F}$ NMR spectrum for 3 (in $\mathrm{CDCl}_{3}$ ).

Data for $\left[\mathrm{K}(\mathrm{DME})_{2}\right]\left[\mathrm{Ce}(\mathrm{TriNOx}) \mathbf{N}\left(\mathbf{3 , 5}-\left(\mathrm{CF}_{3}\right)_{2} \mathbf{C}_{6} \mathbf{H}_{3}\right)\right](4)$. To the solution of 3 (300 mg, $0.33 \mathrm{mmol}, 1.0$ equiv) in fluorobenzene $(4 \mathrm{~mL})$ was added the solution of $\mathrm{KN}\left(\mathrm{SiMe}_{3}\right)_{2}$ (65.5 mg, $0.33 \mathrm{mmol}, 1.0$ equiv) in fluorobenzene $(2 \mathrm{~mL})$. The reaction mixture was stirred overnight and then the volatiles were removed under reduced pressure. The obtained crude solid product was dissolved in $7 \mathrm{~mL}$ of hexanes, filtered over celite and 
DME $(3.0 \mathrm{~mL})$ was added to the filtrate. Cooling the resulting solution to $-25^{\circ} \mathrm{C}$ yielded purple crystals of 4, which were separated by decantation. Yield: $104 \mathrm{mg}, 28 \%$. The lack of paramagnetically shifted resonances for the $t$-Bu groups ${ }^{1}$ in the ${ }^{1} \mathrm{H}$ NMR spectrum of 4 indicates the absence of the TriNOx-Ce $\mathrm{e}^{\mathrm{III}}$ moieties in solution.

${ }^{1} \mathrm{H}$ NMR (300 MHz, THF- $\left.d_{8}\right): \delta 7.95\left(\mathrm{~d},{ }^{2} J_{\mathrm{HH}}=8.2 \mathrm{~Hz}, 3 \mathrm{H}, \mathrm{Ar}-\mathrm{H}\right), 7.35(\mathrm{~m}, 3 \mathrm{H}, \mathrm{Ar}-\mathrm{H})$, $7.08\left(\mathrm{~d},{ }^{2} J_{\mathrm{HH}}=4.5 \mathrm{~Hz}, 6 \mathrm{H}, \mathrm{Ar}-\mathrm{H}\right), 6.09\left(\mathrm{~s}, 2 \mathrm{H}, o-\mathrm{H}, 3,5-\left(\mathrm{CF}_{3}\right)_{2} \mathrm{C}_{6} \mathrm{H}_{3}\right), 5.79(\mathrm{~s}, 1 \mathrm{H}, p-\mathrm{H}$, 3,5- $\left.\left(\mathrm{CF}_{3}\right)_{2} \mathrm{C}_{6} \mathrm{H}_{3}\right), 3.91\left(\mathrm{~d},{ }^{1} J_{\mathrm{HH}}=11.6 \mathrm{~Hz}, 3 \mathrm{H}, \mathrm{CH}_{2}\right), 3.49\left(\mathrm{~s}, 8 \mathrm{H}, \mathrm{CH}_{2}, \mathrm{DME}\right), 3.33$ (s, 12H, $\left.\mathrm{CH}_{3}, \mathrm{DME}\right), 2.24\left(\mathrm{~d},{ }^{1} \mathrm{~J}_{\mathrm{HH}}=11.6 \mathrm{~Hz}, 3 \mathrm{H}, \mathrm{CH}_{2}\right), 1.09(\mathrm{~s}, 27 \mathrm{H}) .{ }^{13} \mathrm{C} \mathrm{NMR}(125.77$ MHz, THF- $\left.d_{8}\right): 152.82,152.61,133.75,132.37,130.39,130.26\left(\mathrm{q}, J_{\mathrm{FC}}=30.1 \mathrm{~Hz}\right)$, 128.28, $127.40(\mathrm{~m}), 127.13$ (q, $\left.J_{\mathrm{FC}}=270.7 \mathrm{~Hz}\right), 125.77,102.29(\mathrm{~m}), 72.75\left(\mathrm{CH}_{2}, \mathrm{DME}\right)$, $63.69\left(\mathrm{CH}_{2}\right.$, TriNOx $), 61.50\left(\mathrm{C}\left(\mathrm{CH}_{3}\right)_{3}\right), 58.89\left(\mathrm{CH}_{3}, \mathrm{DME}\right), 26.66\left(\mathrm{C}_{\left.\left(\mathrm{CH}_{3}\right)_{3}\right) .}{ }^{19} \mathrm{~F} \mathrm{NMR}\right.$ (282.2 MHz, THF- $\left.d_{8}\right): \delta-63.55$ (s). UV-vis (THF, $\left.\lambda_{\max }, \mathrm{nm}\right): 369,510$. Anal. Calc'd for $\mathrm{C}_{49} \mathrm{H}_{68} \mathrm{CeF}_{6} \mathrm{KN}_{5} \mathrm{O}_{7}: \mathrm{C}, 51.98 ; \mathrm{H}, 6.05 ; \mathrm{N}, 6.19$. Found: C, 51.66; H, 6.09; N, 6.20.

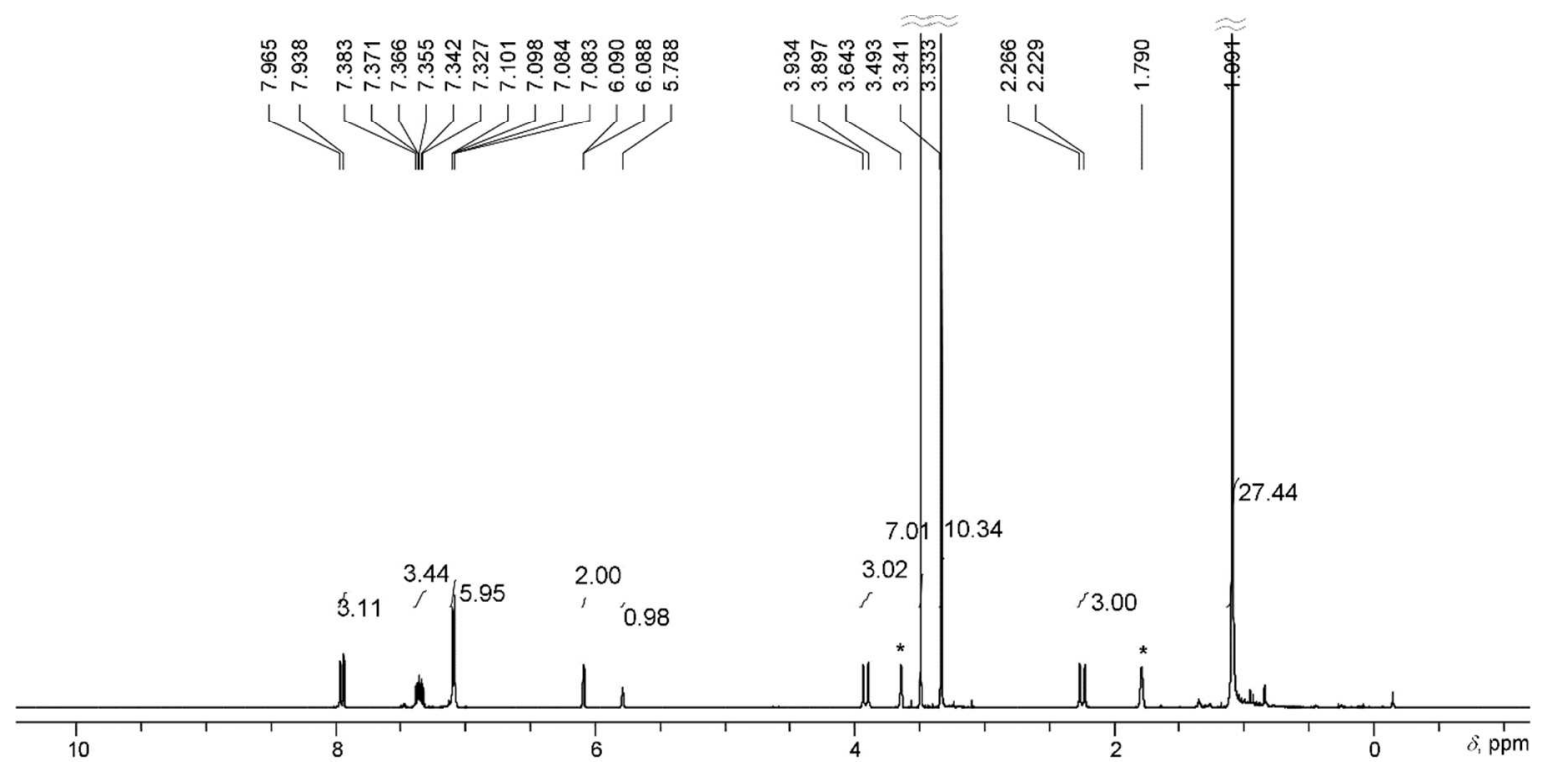

Figure S9. ${ }^{1} \mathrm{H}$ NMR spectrum for 4 (in THF- $d_{8},{ }^{*}$ - proteo solvent signal). 


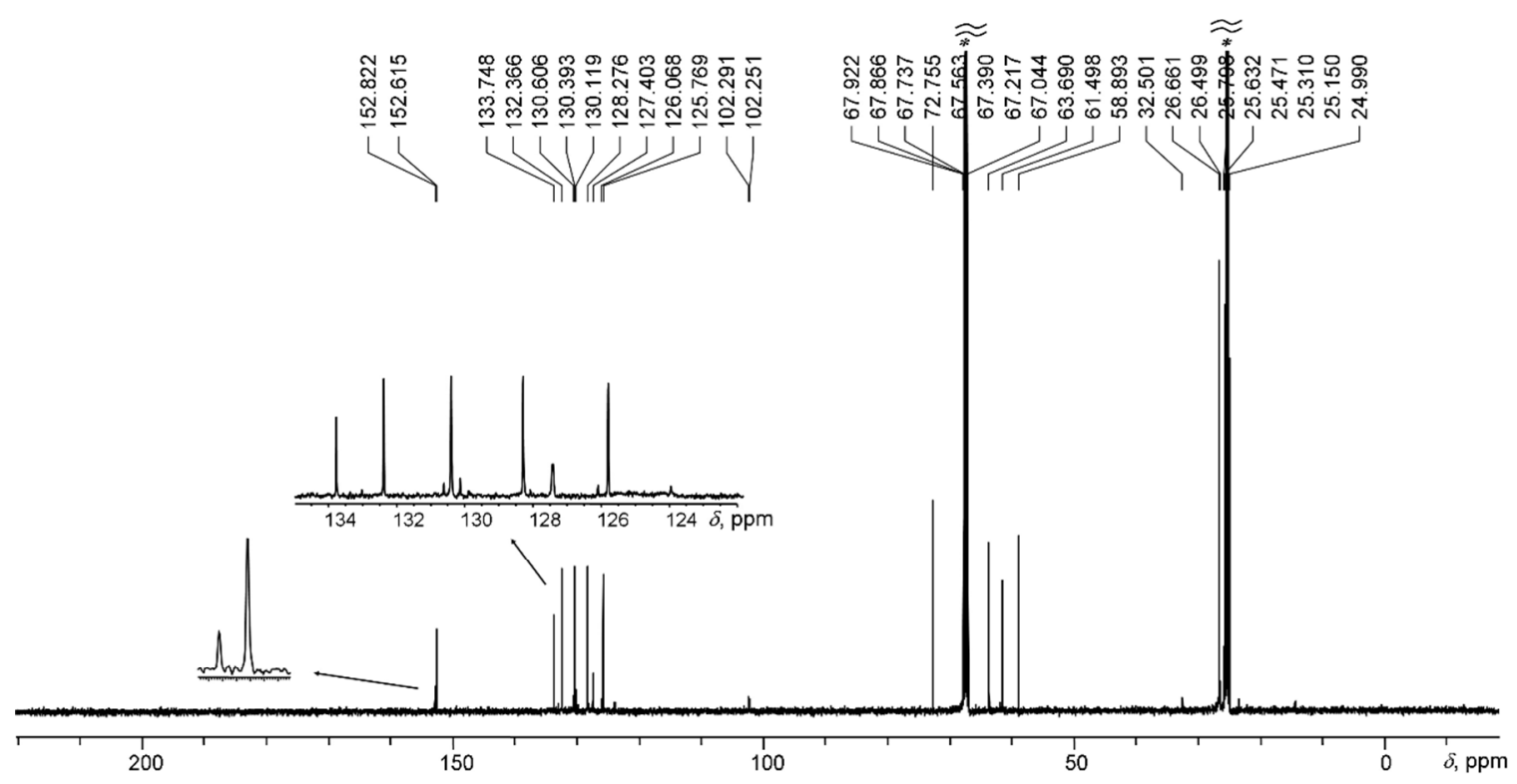

Figure S10. ${ }^{13} \mathrm{C}$ NMR spectrum for 4 (in THF- $d_{8},{ }^{*}$ - solvent signal).

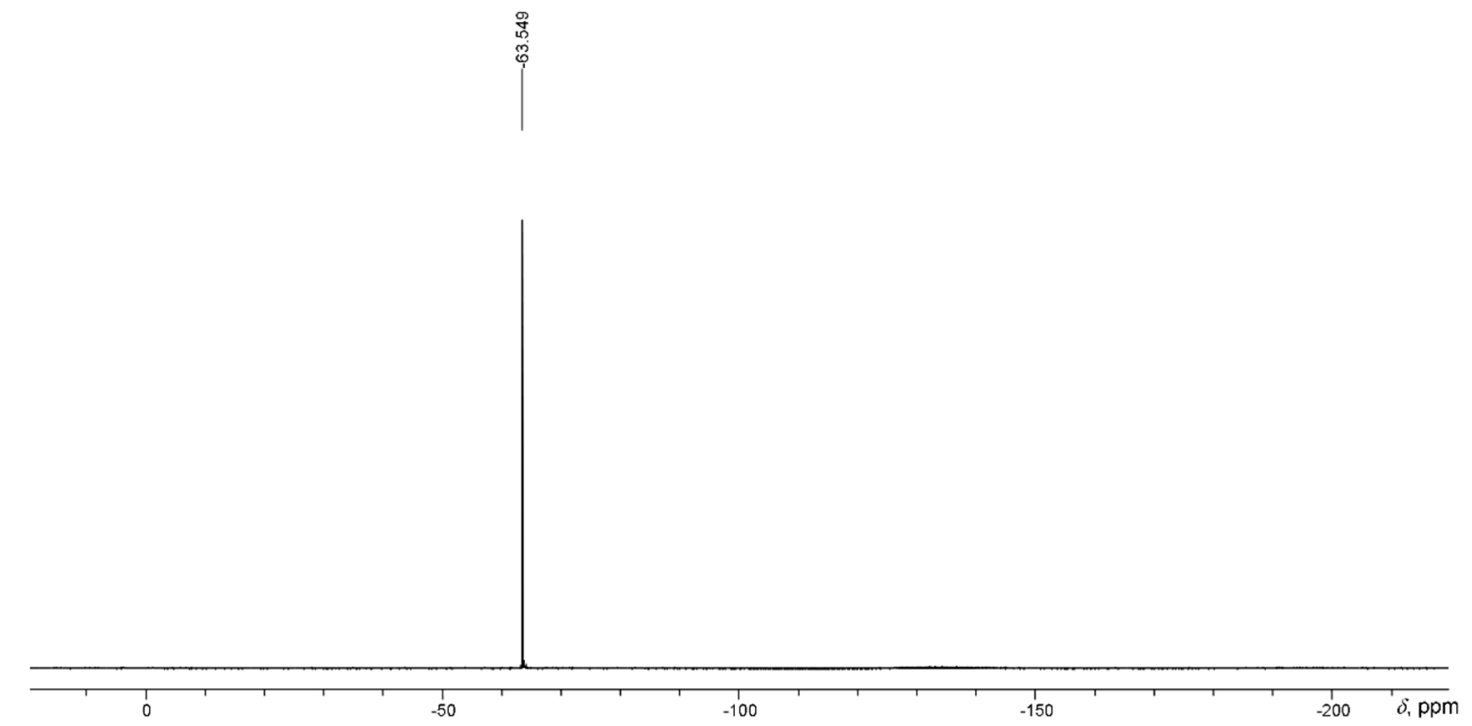

Figure S11. ${ }^{19}$ F NMR spectrum for 4 (in THF- $d_{8}$ ).

\section{Data for Ce(TriNOx)[N(SiMe $\left.)\left(3,5-\left(\mathrm{CF}_{3}\right)_{2} \mathrm{C}_{6} \mathrm{H}_{3}\right)\right](5)$.}

a) Synthesis from 4. To a purple solution of 4 (24 mg, $0.021 \mathrm{mmol}, 1.0$ equiv) in $0.5 \mathrm{~mL}$ of THF- $d_{8}$ was added $5.4 \mu \mathrm{L}\left(0.042 \mathrm{mmol}, 2.0\right.$ equiv) of $\mathrm{Me}_{3} \mathrm{SiCl}$ at $-25^{\circ} \mathrm{C}$ causing a color change to red. The reaction mixture was allowed to warm up to $20{ }^{\circ} \mathrm{C}$. ${ }^{1} \mathrm{H} \mathrm{NMR}$ spectrum for the reaction mixture shows the formation of 5 in the yield of $90 \%$. 
b) Synthesis from 1. To a suspension of 1 ( $229 \mathrm{mg}, 0.30 \mathrm{mmol}, 1.0$ equiv) in $4 \mathrm{~mL}$ of diethyl ether was added solid iodine (46 $\mathrm{mg}, 0.18 \mathrm{mmol}, 0.6$ equiv) and the resulting brown mixture stirred for $2 \mathrm{~h}$. A solution of $\mathrm{KN}\left(3,5-\left(\mathrm{CF}_{3}\right)_{2} \mathrm{C}_{6} \mathrm{H}_{3}\right)\left(\mathrm{SiMe}_{3}\right)(112 \mathrm{mg}, 0.30$ mmol, 1 equiv) in $4 \mathrm{~mL}$ of diethyl ether was then added and the mixture was stirred for additional $3 \mathrm{~h}$. Precipitated KI was removed by filtration over a Celite-packed coarse porosity fritted filter and the solvent and other volatiles were removed under reduced pressure from the filtrate. The resulting red solid product was dried in vacuo and recrystallized upon cooling a hot hexanes solution to $-25^{\circ} \mathrm{C}$. Yield $183 \mathrm{mg}, 62 \%$.

NMR study for $5\left(\mathrm{C}_{6} \mathrm{D}_{6}, 25{ }^{\circ} \mathrm{C}\right)$ shows a restricted rotation about the $\mathrm{N}-\mathrm{C}_{6} \mathrm{H}_{3}\left(3,5-\left(\mathrm{CF}_{3}\right)_{2}\right)$ bond on the NMR time scale.

${ }^{1} \mathrm{H}$ NMR (300 MHz, $\left.\mathrm{C}_{6} \mathrm{D}_{6}\right): \delta 8.49\left(\mathrm{~s}, 1 \mathrm{H},\left(\mathrm{CF}_{3}\right)_{2} \mathrm{C}_{6} \mathrm{H}_{3}\right), 8.02\left(\mathrm{~s}, 1 \mathrm{H},\left(\mathrm{CF}_{3}\right)_{2} \mathrm{C}_{6} \mathrm{H}_{3}\right), 7.65$ (s, $\left.1 \mathrm{H},\left(\mathrm{CF}_{3}\right)_{2} \mathrm{C}_{6} \mathrm{H}_{3}\right), 7.39\left(\mathrm{~d},{ }^{2} J_{\mathrm{HH}}=7.8 \mathrm{~Hz}, 3 \mathrm{H}, \mathrm{Ar}-\mathrm{H}\right), 7.04-6.99(\mathrm{~m}, 3 \mathrm{H}, \mathrm{Ar}-\mathrm{H}), 6.88-6.83$ $(\mathrm{m}, 6 \mathrm{H}, \mathrm{Ar}-\mathrm{H}), 4.30\left(\mathrm{~d},{ }^{1} \mathrm{~J}_{\mathrm{HH}}=11.9 \mathrm{~Hz}, 3 \mathrm{H}, \mathrm{CH}_{2}\right), 2.40\left(\mathrm{~d},{ }^{1} J_{\mathrm{HH}}=11.9 \mathrm{~Hz}, 3 \mathrm{H}, \mathrm{CH}_{2}\right)$, 0.63 (s, 27H, $\left.\mathrm{C}\left(\mathrm{CH}_{3}\right)_{3}\right), 0.62$ (s, 9H, Si( $\left.\left.\mathrm{CH}_{3}\right)_{3}\right) .{ }^{13} \mathrm{C} \mathrm{NMR}\left(125.77 \mathrm{MHz}, \mathrm{THF}-d_{8}\right): 160.00$, $149.36,132.50,131.77,131.22,130.85\left(\mathrm{q}, J_{\mathrm{FC}}=32.9 \mathrm{~Hz}\right), 130.70\left(\mathrm{q}, J_{\mathrm{FC}}=32.5 \mathrm{~Hz}\right)$, $129.81,128.97,128.34,127.87\left(\mathrm{q}, J_{\mathrm{FC}}=272.75 \mathrm{~Hz}\right), 127.74\left(\mathrm{q}, J_{\mathrm{FC}}=272.75 \mathrm{~Hz}\right)$, 126.80, $113.61(\mathrm{~m}), 65.14\left(\mathrm{CH}_{2}\right.$, TriNOx $), 61.34\left(\mathrm{C}_{\left.\left(\mathrm{CH}_{3}\right)_{3}\right),} 26.05\left(\mathrm{C}_{(\mathrm{CH}}\right)_{3}\right), 4.69$ $\left(\mathrm{Si}\left(\mathrm{CH}_{3}\right)_{3}\right) .{ }^{19} \mathrm{~F}$ NMR $\left(282.2 \mathrm{MHz}, \mathrm{C}_{6} \mathrm{D}_{6}\right): \delta-61.57(\mathrm{~s}),-62.25$ (s). UV-vis (THF, $\lambda_{\max }$, nm): 403. Anal. Calc'd for $\mathrm{C}_{44} \mathrm{H}_{57} \mathrm{CeF}_{6} \mathrm{~N}_{5} \mathrm{O}_{3} \mathrm{Si}$ : C, 53.59; H, 5.83; N, 7.10. Found: C, $53.49 ; \mathrm{H}, 6.02 ; \mathrm{N}, 7.04$.
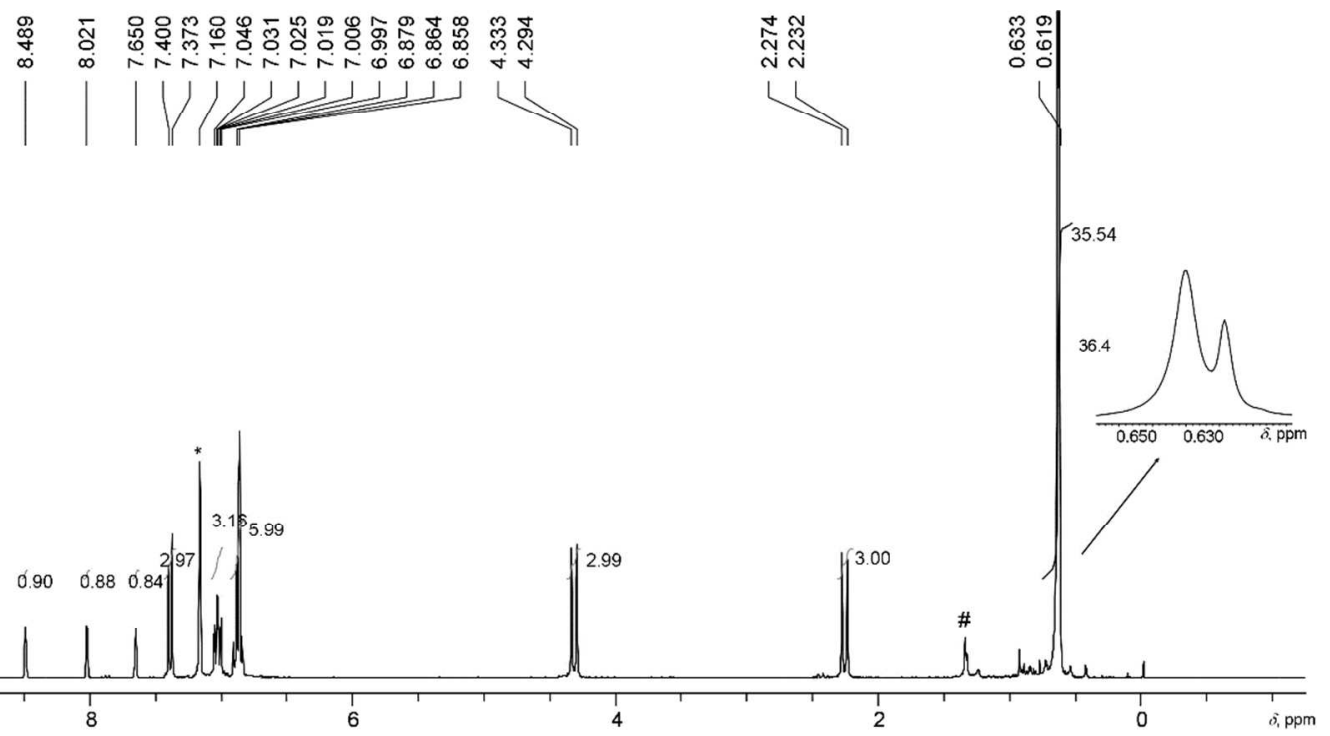

Figure S12. ${ }^{1} \mathrm{H}$ NMR spectrum for 5 (in $\mathrm{C}_{6} \mathrm{D}_{6},{ }^{*}$ - proteo solvent signal, \# - grease). 


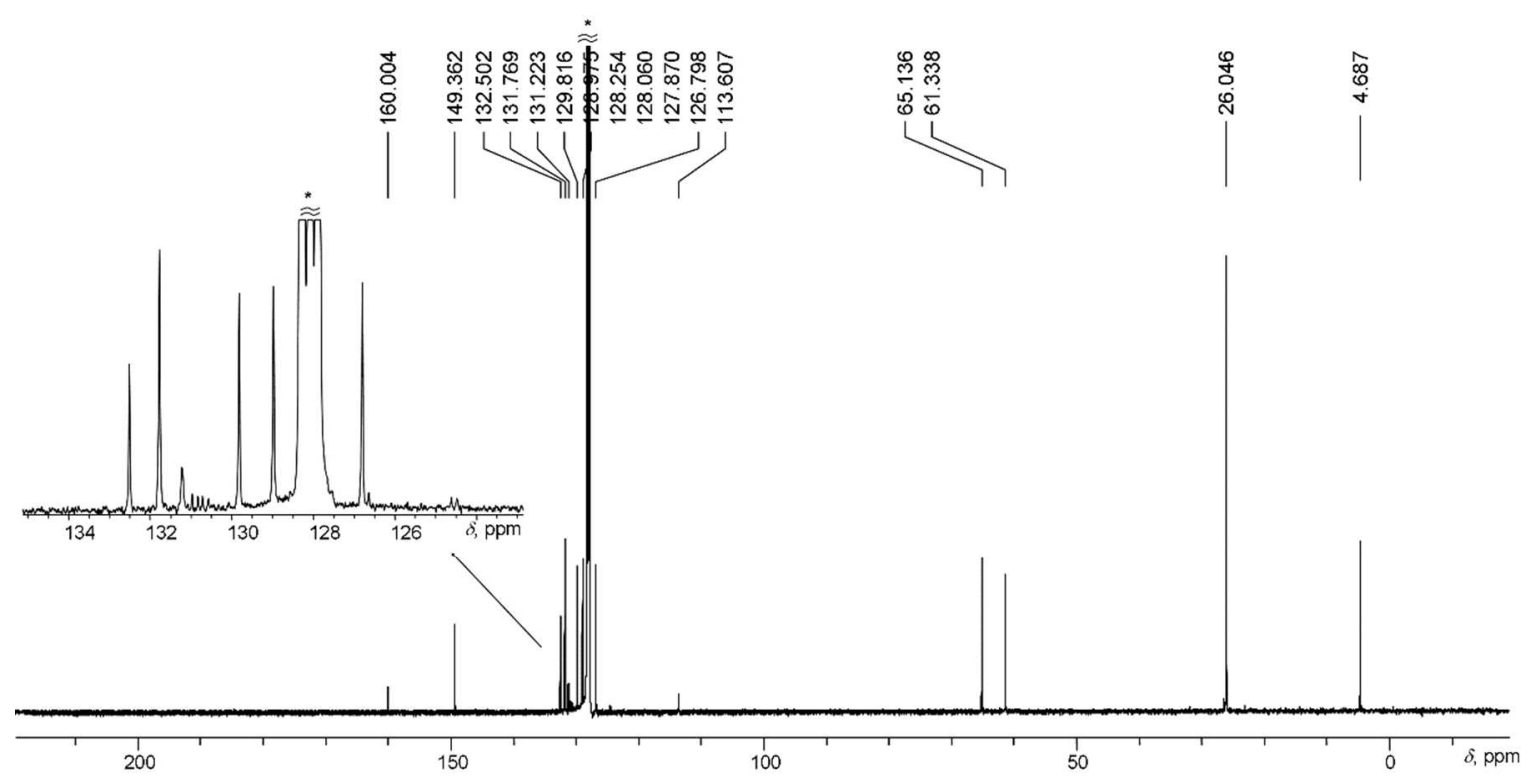

Figure S13. ${ }^{13} \mathrm{C}$ NMR spectrum for 5 (in $\mathrm{C}_{6} \mathrm{D}_{6}$, * - solvent signal).

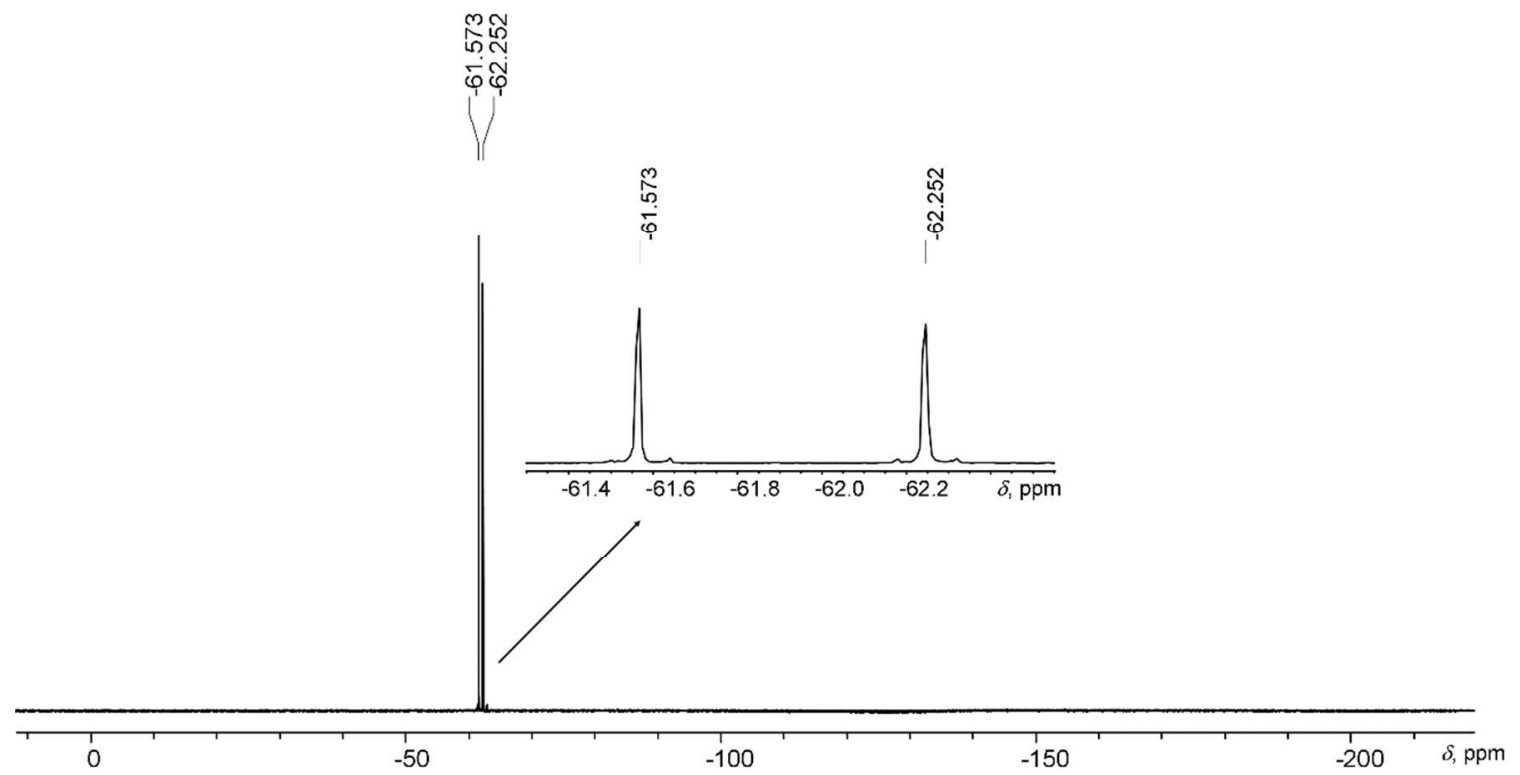

Figure S14. ${ }^{19} \mathrm{~F}$ NMR spectrum for 5 (in $\mathrm{C}_{6} \mathrm{D}_{6}$ ). 


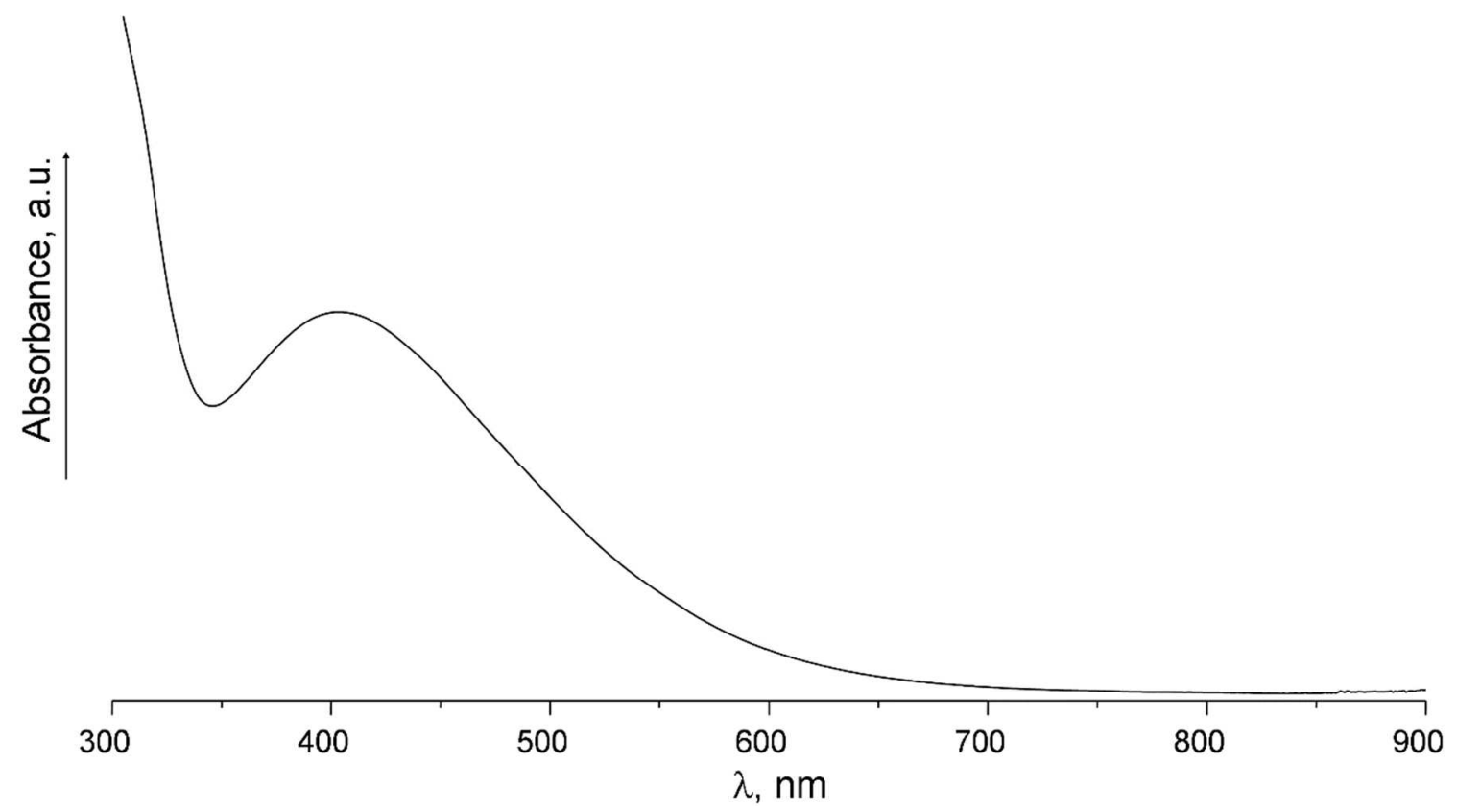

Figure S15. UV-vis spectrum for 5 (in THF).

\section{Crystal Structure Determination and Refinement}

X-ray intensity data were collected on a Bruker APEXII CCD area detector employing graphite-monochromated Mo- $K_{\alpha}$ radiation $(\lambda=0.71073 \AA)$ at $100(1) \mathrm{K}$. Rotation frames were integrated using SAINT, ${ }^{3}$ producing a listing of unaveraged $F^{2}$ and $\sigma\left(F^{2}\right)$ values which were then passed to the SHELXTL program package ${ }^{4}$ for further processing and structure solution. The intensity data were corrected for Lorentz and polarization effects and for absorption using SADABS ${ }^{5}$ or TWINABS. ${ }^{6}$ Structures were solved by direct methods (SHELXS-97). ${ }^{7}$ Refinement was performed by full-matrix least squares based on $F^{2}$ using SHELXL-2014. ${ }^{8}$ All of the reflections were used during refinement. Nonhydrogen atoms were refined anisotropically and hydrogen atoms were refined using a riding model.

The crystal of $\mathbf{3}$ was grown as a non-merohedral two-component twin with one domain rotated from another domain by $180^{\circ}$ degrees about reciprocal axis and real axis. The minor twin component presents in the crystal at 0.422(1).

For 4 there was a region of a disordered intestinal dimetoxyethane molecule for which a reliable disorder model could not be devised; the X-ray data were corrected for the presence of disordered solvent using SQUEEZE. ${ }^{9}$ 
Disorder in 4. The O(6)-DME molecule is positionally disordered over two positions with the major component presents at $0.556(18)$ of time. The $\mathrm{C}(41)$-trifluoromethyl group shows a rotational disorder over two positions with the major component presents at 0.66(4) of time. Disorders were refined with bond restraints.

For further details see Table S1.

Table S1. Crystallographic Data for 2, 3·THF, 4, and 5.

\begin{tabular}{|c|c|c|c|c|}
\hline parameter & 2 & $\mathbf{3} \cdot \mathrm{THF}$ & 4 & 5 \\
\hline formula & $\mathrm{C}_{74} \mathrm{H}_{118} \mathrm{Ce}_{2} \mathrm{~N}_{10} \mathrm{O}_{6} \mathrm{Si}_{4}$ & $\mathrm{C}_{45} \mathrm{H}_{57} \mathrm{CeF}_{6} \mathrm{~N}_{5} \mathrm{O}_{4}$ & $\mathrm{C}_{49} \mathrm{H}_{68} \mathrm{CeN}_{5} \mathrm{O}_{7} \mathrm{~F}_{6} \mathrm{~K}$ & $\mathrm{C}_{44} \mathrm{H}_{57} \mathrm{CeF}_{6} \mathrm{~N}_{5} \mathrm{O}_{3} \mathrm{Si}$ \\
\hline$M_{\mathrm{r}}$ & 1636.38 & 986.07 & 1074.36 & 986.15 \\
\hline$a[\AA]$ & $31.4376(8)$ & $11.9773(3)$ & $11.8007(6)$ & $11.3019(3)$ \\
\hline$b[\AA]$ & $13.3371(4)$ & $17.8482(4)$ & $17.3405(9)$ & $17.3405(9)$ \\
\hline$c[\AA]$ & $19.0763(5)$ & $20.9505(5)$ & $14.0927(7)$ & $18.6065(5)$ \\
\hline$\alpha[\operatorname{deg}]$ & 90 & 90 & 90 & $103.394(2)$ \\
\hline$\beta[\operatorname{deg}]$ & $91.0840(10)$ & $97.3430(10)$ & $91.239(3)$ & $93.797(2)$ \\
\hline$\gamma[\mathrm{deg}]$ & 90 & 90 & 90 & $113.733(2)$ \\
\hline$V\left[\AA^{3}\right]$ & $7997.0(4)$ & $4441.93(18)$ & $2883.1(3)$ & $2247.02(11)$ \\
\hline Z & 4 & 4 & 2 & 2 \\
\hline space group & $P 2_{1} / c$ & $P 2_{1} / n$ & $P 2_{1}$ & $P-1$ \\
\hline$\rho_{\text {calcd }},\left[\mathrm{g} \mathrm{cm}^{-3}\right]$ & 1.359 & 1.475 & 1.304 & 1.458 \\
\hline$\mu\left[\mathrm{mm}^{-1}\right]$ & 1.238 & 1.098 & 0.929 & 1.109 \\
\hline $2 \theta$ range $[\mathrm{deg}]$ & $2.59-55.10$ & $3.73-55.21$ & $3.72-55.12$ & $3.80-55.13$ \\
\hline data collected & 206175 & 102664 & 60529 & 76878 \\
\hline no. unique data, $R_{\text {int }}$ & $18446,0.0898$ & $10235,0.0824$ & $12814,0.0398$ & $10165,0.0312$ \\
\hline no. obsd data $[I \geq 2 \sigma(I)]$ & 18446 & 10235 & 12814 & 8947 \\
\hline$R_{l}(\text { all data })^{[\mathrm{a}]}$ & 0.0847 & 0.0610 & 0.0349 & 0.0341 \\
\hline$w R_{2}$ (all data) $^{[\mathrm{b}]}$ & 0.1050 & 0.0916 & 0.0579 & 0.0534 \\
\hline$S^{[\mathrm{c}]}$ & 1.059 & 1.031 & 1.006 & 1.031 \\
\hline no. of variables & 892 & 559 & 720 & 553 \\
\hline peak/hole $\left[\mathrm{e} \AA^{-3}\right.$ ] & $2.04 /-1.39$ & $1.75 /-0.79$ & $0.94 /-0.42$ & $0.64 /-0.37$ \\
\hline
\end{tabular}

[a] $R_{1}=\Sigma|| F_{\mathrm{o}}|-| F_{\mathrm{c}}|| / 2\left|F_{\mathrm{o}}\right| .{ }^{[\mathrm{b}]} w R_{2}=\left[2\left[w\left(F_{\mathrm{o}}{ }^{2}-F_{\mathrm{c}}\right)^{2}\right] / 2\left[w\left(F_{\mathrm{o}}\right)^{2}\right]\right]^{1 / 2} . \quad[\mathrm{c}]$ Quality-of-fit $\left[2\left[w\left(F_{\mathrm{o}}^{2}-F_{\mathrm{c}}^{2}\right)^{2}\right] /\left(N_{\mathrm{obs}}-N_{\text {params }}\right)\right]^{1 / 2}$, based on all data. 


\section{Computational Details}

Gaussian 09, revision D.01, was used for geometry optimization, vibrational frequencies and electronic structure calculations. ${ }^{10}$ The B3LYP hybrid DFT method was employed, with a 28-electron small core pseudopotential on cerium with published segmented natural orbital basis set incorporating quasi-relativistic effects, ${ }^{11-13}$ and the $6-31 \mathrm{G}^{*}$ basis set on all other atoms. No restraints were imposed other than spin. Molecular orbitals were rendered with the program Chemcraft v1.6 $6^{14}$ at an isovalue of 0.03 . Mayer bond orders and atomic orbital contributions to individual molecular orbitals were calculated with the AOMix program ${ }^{15,16}$ through fragment molecular orbital analysis. One small imaginary frequency of $-2.28 \mathrm{~cm}^{-1}$ was found for $4^{-}$and all other frequency calculations found no imaginary frequencies, confirming that the optimized structures were minima on potential energy surfaces. The NBO 6.0 package was used to obtain natural charges and to determine the orbital contributions. ${ }^{17}$

Table S2. Absolute energies for $\mathbf{3}, \mathbf{4}$ and $\mathbf{4}^{-}$(in Hartrees).

\begin{tabular}{|c|c|}
\hline Complex & B3LYP/6-31G* \\
\hline 3 & -3165.52016840 \\
\hline 4 & -4382.59829862 \\
\hline 4- & -3164.94399354 \\
\hline
\end{tabular}

Table S3. Cartesian coordinates for 3 .

$\begin{array}{llll}\text { Ce } & -0.35591784 & -0.38520617 & -0.00603159 \\ \text { F } & 6.92137505 & -2.90366107 & -1.22513159 \\ \text { F } & 7.66376045 & -2.18364009 & 0.68453018 \\ \text { F } & 6.13189752 & -3.73185820 & 0.61984955 \\ \text { F } & 4.35639719 & 2.99168358 & -0.70956059 \\ \text { F } & 6.43799041 & 2.50319281 & -0.28832090 \\ \text { F } & 5.01918080 & 2.73405281 & 1.34100820 \\ \text { O } & 0.64202310 & 1.42578985 & -0.75192453 \\ \text { O } & -0.13676790 & -0.70090034 & 2.15577429 \\ \text { O } & -0.62761843 & -2.23703119 & -1.17962175 \\ \text { N } & -0.45940499 & 1.70652143 & -1.60008350 \\ \text { N } & -1.01193990 & 0.39036801 & 2.40774778 \\ \text { N } & -1.97473027 & -2.31848632 & -0.74068375\end{array}$




\begin{tabular}{|c|c|c|c|}
\hline $\mathrm{N}$ & -3.22140608 & 0.70146644 & -0.08176228 \\
\hline $\mathrm{N}$ & 1.77685770 & -1.29508163 & -0.21390458 \\
\hline $\mathrm{H}$ & 1.68278923 & -2.30443219 & -0.32927926 \\
\hline $\mathrm{C}$ & -3.35598473 & 1.74914129 & -1.13508688 \\
\hline $\mathrm{H}$ & -4.40828729 & 2.08283986 & -1.17964715 \\
\hline $\mathrm{H}$ & -3.13258481 & 1.26971075 & -2.08661377 \\
\hline $\mathrm{C}$ & -2.47958521 & 2.96330295 & -0.92649209 \\
\hline $\mathrm{C}$ & -3.06193707 & 4.16316203 & -0.48762446 \\
\hline $\mathrm{H}$ & -4.14028741 & 4.20083562 & -0.35176081 \\
\hline $\mathrm{C}$ & -2.29163533 & 5.28855367 & -0.20427026 \\
\hline $\mathrm{H}$ & -2.76791908 & 6.20346747 & 0.13667086 \\
\hline $\mathrm{C}$ & -0.90328285 & 5.21871816 & -0.33731531 \\
\hline $\mathrm{H}$ & -0.28363150 & 6.07678836 & -0.09182699 \\
\hline $\mathrm{C}$ & -0.30688113 & 4.04347477 & -0.78805760 \\
\hline $\mathrm{H}$ & 0.77068175 & 3.97069991 & -0.86481766 \\
\hline $\mathrm{C}$ & -1.08764012 & 2.92674839 & -1.12127520 \\
\hline $\mathrm{C}$ & 0.00565764 & 1.71345465 & -3.05533071 \\
\hline $\mathrm{C}$ & 0.51636800 & 0.30109031 & -3.37803943 \\
\hline $\mathrm{H}$ & -0.26311935 & -0.45193566 & -3.21851352 \\
\hline $\mathrm{H}$ & 1.38507981 & 0.03747845 & -2.76993289 \\
\hline $\mathrm{H}$ & 0.81245856 & 0.25359461 & -4.43182257 \\
\hline $\mathrm{C}$ & 1.13848560 & 2.72959504 & -3.29196949 \\
\hline $\mathrm{H}$ & 1.53554571 & 2.59515457 & -4.30423005 \\
\hline $\mathrm{H}$ & 1.95652279 & 2.57372120 & -2.58325959 \\
\hline $\mathrm{H}$ & 0.78784410 & 3.76304394 & -3.21159916 \\
\hline $\mathrm{C}$ & -1.19118716 & 2.03875308 & -3.96216671 \\
\hline $\mathrm{H}$ & -1.62904246 & 3.01598054 & -3.73563341 \\
\hline $\mathrm{H}$ & -1.97150113 & 1.27383964 & -3.89184104 \\
\hline $\mathrm{H}$ & -0.84945896 & 2.06321441 & -5.00250770 \\
\hline $\mathrm{C}$ & -3.56884026 & 1.29332544 & 1.24294247 \\
\hline $\mathrm{H}$ & -4.59486002 & 1.70114516 & 1.20017415 \\
\hline $\mathrm{H}$ & -2.89803250 & 2.13508501 & 1.40066208 \\
\hline $\mathrm{C}$ & -3.49038609 & 0.31797848 & 2.39716545 \\
\hline $\mathrm{C}$ & -4.68081060 & -0.18537548 & 2.94675993 \\
\hline $\mathrm{H}$ & -5.62902202 & 0.17716540 & 2.55655940 \\
\hline $\mathrm{C}$ & -4.67443102 & -1.14635602 & 3.95497363 \\
\hline $\mathrm{H}$ & -5.61142905 & -1.51945318 & 4.35870906 \\
\hline
\end{tabular}




\begin{tabular}{|c|c|c|c|}
\hline $\mathrm{C}$ & -3.45395836 & -1.64008682 & 4.42029602 \\
\hline $\mathrm{H}$ & -3.42864654 & -2.41032415 & 5.18631733 \\
\hline $\mathrm{C}$ & -2.26127809 & -1.14017554 & 3.90372692 \\
\hline $\mathrm{H}$ & -1.31019029 & -1.53260172 & 4.24050014 \\
\hline $\mathrm{C}$ & -2.26603314 & -0.13630379 & 2.92343320 \\
\hline $\mathrm{C}$ & -0.30711774 & 1.41400017 & 3.30040632 \\
\hline $\mathrm{C}$ & 0.97598220 & 1.86021506 & 2.58521363 \\
\hline $\mathrm{H}$ & 0.76221215 & 2.25585147 & 1.58821630 \\
\hline $\mathrm{H}$ & 1.68872218 & 1.03758277 & 2.49139675 \\
\hline $\mathrm{H}$ & 1.45414986 & 2.65537425 & 3.16768277 \\
\hline $\mathrm{C}$ & 0.06681064 & 0.81419535 & 4.66846073 \\
\hline $\mathrm{H}$ & 0.69168170 & 1.52925495 & 5.21465987 \\
\hline $\mathrm{H}$ & 0.64192532 & -0.10651468 & 4.53650086 \\
\hline $\mathrm{H}$ & -0.81206683 & 0.60451594 & 5.28550567 \\
\hline $\mathrm{C}$ & -1.22758620 & 2.62904841 & 3.49157838 \\
\hline $\mathrm{H}$ & -1.40031061 & 3.15480909 & 2.54700078 \\
\hline $\mathrm{H}$ & -0.74426760 & 3.33466705 & 4.17591566 \\
\hline $\mathrm{H}$ & -2.19221379 & 2.35415080 & 3.93038740 \\
\hline $\mathrm{C}$ & -4.17585307 & -0.40313462 & -0.38439744 \\
\hline $\mathrm{H}$ & -5.20434823 & 0.00055320 & -0.40218732 \\
\hline $\mathrm{H}$ & -4.12263173 & -1.10322411 & 0.44585183 \\
\hline $\mathrm{C}$ & -3.91241865 & -1.10749838 & -1.69668211 \\
\hline $\mathrm{C}$ & -4.74154704 & -0.84192282 & -2.79832494 \\
\hline $\mathrm{H}$ & -5.58395919 & -0.16677267 & -2.66667312 \\
\hline $\mathrm{C}$ & -4.49807214 & -1.40238535 & -4.04994974 \\
\hline $\mathrm{H}$ & -5.15384979 & -1.17559854 & -4.88580786 \\
\hline $\mathrm{C}$ & -3.39081771 & -2.23548388 & -4.22256213 \\
\hline $\mathrm{H}$ & -3.16877583 & -2.65940335 & -5.19808407 \\
\hline $\mathrm{C}$ & -2.56635850 & -2.52749070 & -3.13883922 \\
\hline $\mathrm{H}$ & -1.68954883 & -3.14941850 & -3.26702431 \\
\hline $\mathrm{C}$ & -2.83842875 & -2.00083390 & -1.86718079 \\
\hline $\mathrm{C}$ & -2.20986249 & -3.67811456 & -0.08354422 \\
\hline $\mathrm{C}$ & -1.22511177 & -3.80868544 & 1.08798516 \\
\hline $\mathrm{H}$ & -0.19214094 & -3.86328036 & 0.73649726 \\
\hline $\mathrm{H}$ & -1.44281722 & -4.72911397 & 1.64118959 \\
\hline $\mathrm{H}$ & -1.30979783 & -2.96822650 & 1.78289518 \\
\hline $\mathrm{C}$ & -1.96901680 & -4.83389220 & -1.07226379 \\
\hline
\end{tabular}




$\begin{array}{llll}\mathrm{H} & -0.98105917 & -4.74375756 & -1.53217904 \\ \mathrm{H} & -2.72740608 & -4.86898215 & -1.86016998 \\ \mathrm{H} & -2.00834152 & -5.78584555 & -0.53138952 \\ \mathrm{C} & -3.64665736 & -3.74242395 & 0.45619192 \\ \mathrm{H} & -3.82675987 & -4.74276814 & 0.86460105 \\ \mathrm{H} & -4.39046754 & -3.56930542 & -0.32804843 \\ \mathrm{H} & -3.80610736 & -3.02448201 & 1.26684487 \\ \mathrm{C} & 3.09972862 & -0.94432883 & -0.11095585 \\ \mathrm{C} & 4.14041771 & -1.90534915 & -0.04599582 \\ \mathrm{H} & 3.88705803 & -2.96176073 & -0.06393524 \\ \mathrm{C} & 5.47603423 & -1.52307920 & 0.04668256 \\ \mathrm{C} & 5.84678396 & -0.17608572 & 0.07841186 \\ \mathrm{H} & 6.88700789 & 0.11473736 & 0.14843033 \\ \mathrm{C} & 4.82996457 & 0.78025872 & 0.01058650 \\ \mathrm{C} & 3.49078991 & 0.41747122 & -0.07754373 \\ \mathrm{H} & 2.73008797 & 1.18283037 & -0.16300652 \\ \mathrm{C} & 6.54460846 & -2.58156549 & 0.03707404 \\ \mathrm{C} & 5.16478913 & 2.24421072 & 0.08465596\end{array}$

Table S4. Cartesian coordinates for 4.

$\begin{array}{llll}\text { Ce } & -0.99739200 & -0.41786800 & 0.08532100 \\ \text { K } & 2.37017200 & 1.49589800 & -0.67348000 \\ \text { F } & 5.37434800 & -0.33663300 & 3.17927500 \\ \text { F } & 5.94281600 & -2.43126800 & 3.34526400 \\ \text { F } & 4.08351500 & -1.72751800 & 4.23097200 \\ \text { F } & 5.52041900 & -4.60629600 & -1.00148300 \\ \text { F } & 3.85551600 & -4.13639800 & -2.32327600 \\ \text { F } & 5.54917900 & -2.78181700 & -2.18005800 \\ \text { O } & -1.08706500 & -0.57426500 & 2.31947700 \\ \text { O } & -1.20014700 & -2.26726100 & -1.14724300 \\ \text { O } & -0.32441500 & 1.50543200 & -0.99615000 \\ \mathrm{~N} & -2.10548100 & 0.41075200 & 2.45046800 \\ \mathrm{~N} & -2.52662100 & -2.48721400 & -0.67623500 \\ \mathrm{~N} & -1.53665100 & 1.55771500 & -1.74683400 \\ \mathrm{~N} & -4.23783100 & 0.35409000 & -0.09954500 \\ \mathrm{~N} & 1.03039300 & -0.80908600 & 0.21085600 \\ \mathrm{C} & -4.70340400 & 0.95354300 & 1.17555000\end{array}$




\begin{tabular}{|c|c|c|c|}
\hline $\mathrm{H}$ & -5.77038700 & 1.23582600 & 1.08409100 \\
\hline $\mathrm{H}$ & -4.14264700 & 1.87517200 & 1.31538300 \\
\hline $\mathrm{C}$ & -4.55356700 & 0.04621600 & 2.37687000 \\
\hline $\mathrm{C}$ & -5.69464900 & -0.57303200 & 2.91178200 \\
\hline $\mathrm{H}$ & -6.66446100 & -0.34061300 & 2.47741700 \\
\hline $\mathrm{C}$ & -5.60985800 & -1.49078400 & 3.95613300 \\
\hline $\mathrm{H}$ & -6.50946500 & -1.95880300 & 4.34652200 \\
\hline $\mathrm{C}$ & -4.35485100 & -1.82184000 & 4.47195200 \\
\hline $\mathrm{H}$ & -4.26415400 & -2.55842800 & 5.26616200 \\
\hline $\mathrm{C}$ & -3.21263100 & -1.20455400 & 3.96845500 \\
\hline $\mathrm{H}$ & -2.23188800 & -1.47418200 & 4.33927100 \\
\hline $\mathrm{C}$ & -3.30114200 & -0.24150600 & 2.95074400 \\
\hline $\mathrm{C}$ & -1.58130700 & 1.54781400 & 3.31602800 \\
\hline $\mathrm{C}$ & -0.38349300 & 2.16283500 & 2.57637500 \\
\hline $\mathrm{H}$ & 0.42933700 & 1.43989300 & 2.47480000 \\
\hline $\mathrm{H}$ & -0.00920200 & 3.02723500 & 3.13838900 \\
\hline $\mathrm{H}$ & -0.67277000 & 2.50554600 & 1.57663900 \\
\hline $\mathrm{C}$ & -1.11596200 & 1.05456900 & 4.69986200 \\
\hline $\mathrm{H}$ & -1.95605900 & 0.73661100 & 5.32487300 \\
\hline $\mathrm{H}$ & -0.60113900 & 1.86643800 & 5.22711800 \\
\hline $\mathrm{H}$ & -0.42131700 & 0.21803500 & 4.58642700 \\
\hline $\mathrm{C}$ & -2.66723500 & 2.62212600 & 3.48594500 \\
\hline $\mathrm{H}$ & -2.93077900 & 3.08478500 & 2.52959600 \\
\hline $\mathrm{H}$ & -2.28574300 & 3.41383300 & 4.14090800 \\
\hline $\mathrm{H}$ & -3.57475700 & 2.22107800 & 3.94779600 \\
\hline $\mathrm{C}$ & -5.00983600 & -0.88427300 & -0.37226200 \\
\hline $\mathrm{H}$ & -6.08998200 & -0.64495900 & -0.41649800 \\
\hline $\mathrm{H}$ & -4.86629100 & -1.53982800 & 0.48302600 \\
\hline $\mathrm{C}$ & -4.62959000 & -1.59154000 & -1.65519000 \\
\hline $\mathrm{C}$ & -5.48849600 & -1.49051500 & -2.76249500 \\
\hline $\mathrm{H}$ & -6.42005200 & -0.94085900 & -2.64711600 \\
\hline $\mathrm{C}$ & -5.16889300 & -2.05070300 & -3.99680500 \\
\hline $\mathrm{H}$ & -5.85306100 & -1.95251400 & -4.83530900 \\
\hline $\mathrm{C}$ & -3.95037800 & -2.71655300 & -4.14598700 \\
\hline $\mathrm{H}$ & -3.66900000 & -3.14010700 & -5.10678800 \\
\hline $\mathrm{C}$ & -3.09263100 & -2.84398800 & -3.05698400 \\
\hline $\mathrm{H}$ & -2.13445900 & -3.33583700 & -3.16557900 \\
\hline
\end{tabular}




\begin{tabular}{|c|c|c|c|}
\hline $\mathrm{C}$ & -3.43340700 & -2.32183000 & -1.79839800 \\
\hline $\mathrm{C}$ & -2.58346400 & -3.84571900 & 0.01708400 \\
\hline $\mathrm{C}$ & -1.56986500 & -3.81748600 & 1.17193700 \\
\hline $\mathrm{H}$ & -0.54519100 & -3.75484500 & 0.79812000 \\
\hline $\mathrm{H}$ & -1.66394300 & -4.73842400 & 1.75916600 \\
\hline $\mathrm{H}$ & -1.74977000 & -2.97030100 & 1.84130000 \\
\hline $\mathrm{C}$ & -2.21614100 & -4.99595900 & -0.93983700 \\
\hline $\mathrm{H}$ & -1.25734100 & -4.79186700 & -1.42421100 \\
\hline $\mathrm{H}$ & -2.97855500 & -5.15613400 & -1.70884000 \\
\hline $\mathrm{H}$ & -2.12002600 & -5.92676500 & -0.36924700 \\
\hline $\mathrm{C}$ & -3.99101600 & -4.07177100 & 0.58980700 \\
\hline $\mathrm{H}$ & -4.03525900 & -5.06925600 & 1.04124400 \\
\hline $\mathrm{H}$ & -4.76480100 & -4.02684300 & -0.18371500 \\
\hline $\mathrm{H}$ & -4.22661300 & -3.34542700 & 1.37394700 \\
\hline $\mathrm{C}$ & -4.44730300 & 1.32259800 & -1.20039700 \\
\hline $\mathrm{H}$ & -5.52373500 & 1.57142600 & -1.27728600 \\
\hline $\mathrm{H}$ & -4.17546200 & 0.81659700 & -2.12284600 \\
\hline $\mathrm{C}$ & -3.67494100 & 2.61886800 & -1.06114000 \\
\hline $\mathrm{C}$ & -4.36408000 & 3.77412500 & -0.65588300 \\
\hline $\mathrm{H}$ & -5.43447200 & 3.70201800 & -0.47699300 \\
\hline $\mathrm{C}$ & -3.71667000 & 4.99164800 & -0.46187400 \\
\hline $\mathrm{H}$ & -4.27898300 & 5.86502100 & -0.14306000 \\
\hline $\mathrm{C}$ & -2.33777100 & 5.06827700 & -0.66761200 \\
\hline $\mathrm{H}$ & -1.81025800 & 6.00591800 & -0.50946900 \\
\hline $\mathrm{C}$ & -1.63651300 & 3.94006000 & -1.08432300 \\
\hline $\mathrm{H}$ & -0.56502800 & 3.98464900 & -1.23352900 \\
\hline $\mathrm{C}$ & -2.29154300 & 2.71874600 & -1.31171600 \\
\hline $\mathrm{C}$ & -1.21799300 & 1.50768800 & -3.23444900 \\
\hline $\mathrm{C}$ & -0.46477900 & 0.19542400 & -3.50073200 \\
\hline $\mathrm{H}$ & 0.50283100 & 0.17658600 & -2.99397600 \\
\hline $\mathrm{H}$ & -0.29529500 & 0.08185700 & -4.57821900 \\
\hline $\mathrm{H}$ & -1.03629500 & -0.67054700 & -3.15465100 \\
\hline $\mathrm{C}$ & -0.35321300 & 2.70122000 & -3.68959800 \\
\hline $\mathrm{H}$ & -0.90641300 & 3.64487000 & -3.65385400 \\
\hline $\mathrm{H}$ & -0.03257000 & 2.54545100 & -4.72649800 \\
\hline $\mathrm{H}$ & 0.53977500 & 2.80170000 & -3.06566400 \\
\hline $\mathrm{C}$ & -2.52253900 & 1.49575200 & -4.04853500 \\
\hline
\end{tabular}




\begin{tabular}{|c|c|c|c|}
\hline $\mathrm{H}$ & -3.09847100 & 0.58302600 & -3.87137200 \\
\hline $\mathrm{H}$ & -2.27548000 & 1.52480600 & -5.11584500 \\
\hline $\mathrm{H}$ & -3.15170700 & 2.36518900 & -3.83320300 \\
\hline $\mathrm{C}$ & 2.19373200 & -1.43856300 & 0.43925900 \\
\hline $\mathrm{C}$ & 2.91857900 & -1.29327000 & 1.66542900 \\
\hline $\mathrm{H}$ & 2.45723100 & -0.71184200 & 2.45842900 \\
\hline $\mathrm{C}$ & 4.16090300 & -1.89279400 & 1.86159000 \\
\hline $\mathrm{C}$ & 4.76371900 & -2.67733600 & 0.87385100 \\
\hline $\mathrm{H}$ & 5.72528400 & -3.14602900 & 1.03791000 \\
\hline $\mathrm{C}$ & 4.06563600 & -2.85242300 & -0.33067500 \\
\hline $\mathrm{C}$ & 2.82658500 & -2.26323300 & -0.55048500 \\
\hline $\mathrm{H}$ & 2.30106800 & -2.41770700 & -1.48701100 \\
\hline $\mathrm{C}$ & 4.88347100 & -1.62020200 & 3.14817200 \\
\hline $\mathrm{C}$ & 4.73255800 & -3.60291700 & -1.44767500 \\
\hline $\mathrm{C}$ & 3.54180300 & 2.66082800 & 2.94701900 \\
\hline $\mathrm{H}$ & 4.50345300 & 3.16957500 & 3.11234200 \\
\hline $\mathrm{H}$ & 3.71713500 & 1.58592000 & 2.89454300 \\
\hline $\mathrm{H}$ & 2.87362700 & 2.88324800 & 3.79116600 \\
\hline $\mathrm{O}$ & 2.95384700 & 3.06391400 & 1.71626500 \\
\hline $\mathrm{C}$ & 2.79689100 & 4.46934500 & 1.62408400 \\
\hline $\mathrm{H}$ & 3.78365700 & 4.95518200 & 1.54818200 \\
\hline $\mathrm{H}$ & 2.30608000 & 4.86466300 & 2.52869500 \\
\hline $\mathrm{C}$ & 1.91195300 & 4.80096700 & 0.42973200 \\
\hline $\mathrm{H}$ & 0.93682000 & 4.32526700 & 0.56629000 \\
\hline $\mathrm{H}$ & 1.76316300 & 5.89171900 & 0.38425800 \\
\hline $\mathrm{O}$ & 2.40286700 & 4.31694700 & -0.81473600 \\
\hline $\mathrm{C}$ & 3.45615900 & 5.08687700 & -1.37623200 \\
\hline $\mathrm{H}$ & 3.14749700 & 6.13438100 & -1.50985400 \\
\hline $\mathrm{H}$ & 3.67975000 & 4.65007200 & -2.35181200 \\
\hline $\mathrm{H}$ & 4.36671400 & 5.05708100 & -0.76285500 \\
\hline $\mathrm{H}$ & 5.79791200 & 0.93915700 & 1.06987300 \\
\hline $\mathrm{O}$ & 5.35382300 & 1.65738100 & -0.84790500 \\
\hline $\mathrm{H}$ & 5.17260800 & -0.40719100 & -1.03268600 \\
\hline $\mathrm{H}$ & 5.73164000 & 1.37208500 & -3.47205300 \\
\hline $\mathrm{C}$ & 6.04160100 & 1.76654600 & 0.39044400 \\
\hline $\mathrm{H}$ & 7.13098900 & 1.78703400 & 0.23347600 \\
\hline $\mathrm{H}$ & 5.72689100 & 2.70811100 & 0.84544700 \\
\hline
\end{tabular}




$\begin{array}{llll}\mathrm{C} & 5.65511200 & 0.44451400 & -1.52875700 \\ \mathrm{H} & 6.74192800 & 0.26399900 & -1.52738600 \\ \mathrm{C} & 5.20395000 & 0.54514500 & -2.97001900 \\ \mathrm{H} & 5.46358500 & -0.39554300 & -3.47831000 \\ \mathrm{O} & 3.80108000 & 0.76517200 & -3.03641800 \\ \mathrm{C} & 3.33649900 & 0.80155300 & -4.37619000 \\ \mathrm{H} & 3.82250300 & 1.61096000 & -4.94296100 \\ \mathrm{H} & 2.26089100 & 0.97962700 & -4.34415300 \\ \mathrm{H} & 3.52772600 & -0.15246200 & -4.88912100\end{array}$

Table S5. Cartesian coordinates for $\mathbf{4}^{-}$.

$\begin{array}{llll}\mathrm{Ce} & 0.03248933 & -0.03260755 & -0.01709396 \\ \mathrm{~F} & -6.57527524 & -2.87630793 & 1.24197725 \\ \mathrm{~F} & -7.48208623 & -2.41034283 & -0.67310963 \\ \mathrm{~F} & -5.62050735 & -3.53687214 & -0.59170948 \\ \mathrm{~F} & -6.46880314 & 2.86240117 & 1.29135032 \\ \mathrm{~F} & -7.39364986 & 2.46001759 & -0.62960569 \\ \mathrm{~F} & -5.49312692 & 3.51809263 & -0.53305814 \\ \mathrm{O} & 0.01267067 & 2.23710351 & -0.02018822 \\ \mathrm{O} & 0.10753271 & -1.14622791 & -1.98991370 \\ \mathrm{O} & 0.05787561 & -1.13028535 & 1.96885916 \\ \mathrm{~N} & 1.20704530 & 2.34626123 & 0.74779686 \\ \mathrm{~N} & 1.31020004 & -0.51970038 & -2.42710326 \\ \mathrm{~N} & 1.26824423 & -1.82389471 & 1.68414556 \\ \mathrm{~N} & 3.53880830 & 0.02372838 & 0.02819938 \\ \mathrm{~N} & -1.99530184 & -0.07893564 & -0.05449845 \\ \mathrm{C} & 3.98473098 & 1.28953021 & 0.65026318 \\ \mathrm{H} & 5.09192980 & 1.30648316 & 0.71996631 \\ \mathrm{H} & 3.60359377 & 1.29335370 & 1.66865628 \\ \mathrm{C} & 3.54000521 & 2.53199008 & -0.09232614 \\ \mathrm{C} & 4.48029302 & 3.22383252 & -0.87390076 \\ \mathrm{H} & 5.51081890 & 2.87417521 & -0.87233923 \\ \mathrm{C} & 4.12393907 & 4.31200445 & -1.66645251 \\ \mathrm{H} & 4.87396406 & 4.82210173 & -2.26592765 \\ \mathrm{C} & 2.78641888 & 4.71351038 & -1.70422057 \\ \mathrm{H} & 2.47804263 & 5.53998662 & -2.34045918 \\ \mathrm{C} & 1.84225629 & 4.05448710 & -0.92239707 \\ & & & \\ \mathrm{H} & & \\ \mathrm{H} & & \end{array}$




\begin{tabular}{|c|c|c|c|}
\hline $\mathrm{H}$ & 0.79678822 & 4.33188801 & -0.96377181 \\
\hline $\mathrm{C}$ & 2.20766121 & 2.99225360 & -0.07739527 \\
\hline $\mathrm{C}$ & 0.88113252 & 3.06984358 & 2.04847493 \\
\hline $\mathrm{C}$ & -0.18454389 & 2.23895509 & 2.78170654 \\
\hline $\mathrm{H}$ & 0.14499367 & 1.20505466 & 2.92584425 \\
\hline $\mathrm{H}$ & -1.12471701 & 2.22537064 & 2.22540815 \\
\hline $\mathrm{H}$ & -0.37393805 & 2.67782520 & 3.76927522 \\
\hline $\mathrm{C}$ & 0.32059154 & 4.48352162 & 1.79680561 \\
\hline $\mathrm{H}$ & -0.04968931 & 4.90579552 & 2.73871848 \\
\hline $\mathrm{H}$ & -0.51279821 & 4.42961965 & 1.09131774 \\
\hline $\mathrm{H}$ & 1.08016486 & 5.16604586 & 1.40084180 \\
\hline $\mathrm{C}$ & 2.13894517 & 3.15489351 & 2.92745922 \\
\hline $\mathrm{H}$ & 2.96308474 & 3.66880604 & 2.42120952 \\
\hline $\mathrm{H}$ & 2.47657316 & 2.16115560 & 3.23846009 \\
\hline $\mathrm{H}$ & 1.90350858 & 3.72261559 & 3.83551871 \\
\hline $\mathrm{C}$ & 4.04705952 & -0.06832800 & -1.35783094 \\
\hline $\mathrm{H}$ & 5.15542916 & -0.01649596 & -1.35579179 \\
\hline $\mathrm{H}$ & 3.69136569 & 0.81155987 & -1.88860308 \\
\hline $\mathrm{C}$ & 3.63583718 & -1.33141925 & -2.08471291 \\
\hline $\mathrm{C}$ & 4.58768508 & -2.34964732 & -2.26069061 \\
\hline $\mathrm{H}$ & 5.60419334 & -2.17248749 & -1.91499851 \\
\hline $\mathrm{C}$ & 4.25861477 & -3.57743504 & -2.82940684 \\
\hline $\mathrm{H}$ & 5.01665754 & -4.34821779 & -2.94509681 \\
\hline $\mathrm{C}$ & 2.93672352 & -3.81305482 & -3.21489656 \\
\hline $\mathrm{H}$ & 2.64840026 & -4.77567875 & -3.63105041 \\
\hline $\mathrm{C}$ & 1.98214870 & -2.81124704 & -3.06646571 \\
\hline $\mathrm{H}$ & 0.94699160 & -2.98885483 & -3.32804807 \\
\hline $\mathrm{C}$ & 2.32193134 & -1.55053135 & -2.54581467 \\
\hline $\mathrm{C}$ & 1.01766301 & 0.24832161 & -3.70919910 \\
\hline $\mathrm{C}$ & -0.05570178 & 1.29864626 & -3.37922626 \\
\hline $\mathrm{H}$ & 0.25881876 & 1.94553249 & -2.55422692 \\
\hline $\mathrm{H}$ & -0.99930714 & 0.82291011 & -3.10156486 \\
\hline $\mathrm{H}$ & -0.23245523 & 1.92986815 & -4.25907031 \\
\hline $\mathrm{C}$ & 0.48419165 & -0.67163456 & -4.82500261 \\
\hline $\mathrm{H}$ & 0.13408546 & -0.06308013 & -5.66737541 \\
\hline $\mathrm{H}$ & -0.35879469 & -1.25752416 & -4.44909262 \\
\hline $\mathrm{H}$ & 1.25373457 & -1.35312522 & -5.20308807 \\
\hline
\end{tabular}




\begin{tabular}{|c|c|c|c|}
\hline $\mathrm{C}$ & 2.28912471 & 0.96708410 & -4.18724755 \\
\hline $\mathrm{H}$ & 2.61011072 & 1.72993329 & -3.47089517 \\
\hline $\mathrm{H}$ & 2.07849302 & 1.47385811 & -5.13655081 \\
\hline $\mathrm{H}$ & 3.11701325 & 0.27134838 & -4.36136523 \\
\hline $\mathrm{C}$ & 4.02157099 & -1.13010887 & 0.81829455 \\
\hline $\mathrm{H}$ & 5.13049026 & -1.16240501 & 0.79829487 \\
\hline $\mathrm{H}$ & 3.66912355 & -2.02704668 & 0.31444111 \\
\hline $\mathrm{C}$ & 3.57853649 & -1.12082712 & 2.26599743 \\
\hline $\mathrm{C}$ & 4.50893314 & -0.76134459 & 3.25533017 \\
\hline $\mathrm{H}$ & 5.53314733 & -0.55441315 & 2.95138299 \\
\hline $\mathrm{C}$ & 4.14905810 & -0.63168331 & 4.59432528 \\
\hline $\mathrm{H}$ & 4.89076433 & -0.34412127 & 5.33543459 \\
\hline $\mathrm{C}$ & 2.81795974 & -0.84299214 & 4.96198646 \\
\hline $\mathrm{H}$ & 2.50624529 & -0.71590244 & 5.99621589 \\
\hline $\mathrm{C}$ & 1.88432633 & -1.21937804 & 4.00077236 \\
\hline $\mathrm{H}$ & 0.84280777 & -1.35513592 & 4.26260979 \\
\hline $\mathrm{C}$ & 2.25522313 & -1.40600803 & 2.65807260 \\
\hline $\mathrm{C}$ & 0.97759436 & -3.31866129 & 1.65850188 \\
\hline $\mathrm{C}$ & -0.05848429 & -3.56094878 & 0.54898585 \\
\hline $\mathrm{H}$ & -1.00933026 & -3.07891035 & 0.78864835 \\
\hline $\mathrm{H}$ & -0.23438589 & -4.63810310 & 0.43884262 \\
\hline $\mathrm{H}$ & 0.29065079 & -3.17309667 & -0.41357182 \\
\hline $\mathrm{C}$ & 0.39898214 & -3.81735387 & 2.99720701 \\
\hline $\mathrm{H}$ & -0.45458948 & -3.19766054 & 3.28463644 \\
\hline $\mathrm{H}$ & 1.14228235 & -3.79841498 & 3.80151071 \\
\hline $\mathrm{H}$ & 0.05359413 & -4.85227682 & 2.88644162 \\
\hline $\mathrm{C}$ & 2.25998821 & -4.09591816 & 1.32142266 \\
\hline $\mathrm{H}$ & 2.04405441 & -5.17049996 & 1.35086956 \\
\hline $\mathrm{H}$ & 3.06399059 & -3.90046502 & 2.03916776 \\
\hline $\mathrm{H}$ & 2.61704654 & -3.85946424 & 0.31414367 \\
\hline $\mathrm{C}$ & -3.33664856 & -0.05479143 & -0.06128241 \\
\hline $\mathrm{C}$ & -4.12310419 & -1.24945038 & -0.07740142 \\
\hline $\mathrm{H}$ & -3.59947957 & -2.19944690 & -0.09467812 \\
\hline $\mathrm{C}$ & -5.51318226 & -1.21262039 & -0.08398185 \\
\hline $\mathrm{C}$ & -6.21588366 & -0.00119669 & -0.07532429 \\
\hline $\mathrm{H}$ & -7.29817094 & 0.01950850 & -0.09805734 \\
\hline $\mathrm{C}$ & -5.46828009 & 1.18345312 & -0.06317683 \\
\hline
\end{tabular}




$\begin{array}{llll}\text { C } & -4.07785996 & 1.16925430 & -0.05582414 \\ \mathrm{H} & -3.51701113 & 2.09809109 & -0.05511949 \\ \mathrm{C} & -6.28596054 & -2.49729767 & -0.03281972 \\ \mathrm{C} & -6.19434885 & 2.49418786 & 0.01004030\end{array}$

\section{References}

(1) Bogart, J. A.; Lippincott, C. A.; Carroll, P. J.; Booth, C. H.; Schelter, E. J. Chem. Eur. J. 2015, 21, 17850.

(2) Sarazin, Y.; Roșca, D.; Poirier, V.; Roisnel, T.; Silvestru, A.; Maron, L.; Carpentier, J.-F. Organometallics 2010, 29, 6569.

(3) Bruker SAINT; Bruker AXS inc., Madison, Wisconsin, USA, 2009.

(4) Bruker SHELXTL; Bruker AXS Inc., Madison, Wisconsin, USA 2009.

(5) Sheldrick, G. M. SADABS; University of Gottingen, Germany, 2007.

(6) Sheldrick, G. M. TWINABS; University of Gottingen, Germany, 2012.

(7) Sheldrick, G. M. Acta Cryst. 2008, A64, 112.

(8) Sheldrick, G. M. SHELXL 2014/7; University of Gottingen, Germany, 2014.

(9) van der Sluis, P.; Spek, A. L. Acta Cryst. 1990, A46, 194.

(10) Frisch, M. J.; Trucks, G. W.; Schlegel, H. B.; Scuseria, G. E.; Robb, M. A.; Cheeseman, J. R.; Scalmani, G.; Barone, V.; Mennucci, B.; Petersson, G. A.; Nakatsuji, H.; Caricato, M.; Li, X.; Hratchian, H. P.; Izmaylov, A. F.; Bloino, J.; Zheng, G.; Sonnenberg, J. L.; Hada, M.; Ehara, M.; Toyota, K.; Fukuda, R.; Hasegawa, J.; Ishida, M.; Nakajima, T.; Honda, Y.; Kitao, O.; Nakai, H.; Vreven, T.; Montgomery Jr., J. A.; Peralta, J. E.; Ogliaro, F.; Bearpark, M. J.; Heyd, J.; Brothers, E. N.; Kudin, K. N.; Staroverov, V. N.; Kobayashi, R.; Normand, J.; Raghavachari, K.; Rendell, A. P.; Burant, J. C.; Iyengar, S. S.; Tomasi, J.; Cossi, M.; Rega, N.;

Millam, N. J.; Klene, M.; Knox, J. E.; Cross, J. B.; Bakken, V.; Adamo, C.; Jaramillo, J.; Gomperts, R.; Stratmann, R. E.; Yazyev, O.; Austin, A. J.; Cammi, R.; Pomelli, C.; Ochterski, J. W.; Martin, R. L.; Morokuma, K.; Zakrzewski, V. G.; Voth, G. A.; Salvador, P.; Dannenberg, J. J.; Dapprich, S.; Daniels, A. D.; Farkas, Ö.; Foresman, J. B.; Ortiz, J. V.; Cioslowski, J.; Fox, D. J.; Gaussian, Inc.: Wallingford, CT, USA, 2009.

(11) Cao, X.; Dolg, M. J. Mol. Struct. (Theochem) 2002, 581, 139.

(12) Dolg, M.; Stoll, H.; Preuss, H. J. Chem. Phys. 1989, 90, 1730.

(13) Institute for Theoretical Chemistry, University of Cologne, http://www.tc.unikoeln.de/PP/clickpse.en.html.

(14) Chemcraft, www.chemcraftprog.com.

(15) Gorelsky, S. I.; Lever, A. B. P. J. Organomet. Chem. 2001, 635, 187.

(16) Gorelsky, S. I. AOMix: Program for Molecular Orbital Analysis, http://www.sgchem.net/, University of Ottawa, version 6.5, 2011.

(17) Glendening, E. D.; Badenhoop, J. K.; Reed, A. E.; Carpenter, J. E.; Bohmann, J. A.; Morales, C. M.; Weinhold, F. NBO 6.0 2001, University of Wisconsin, Madison. 